\title{
Uncovering Clinical Risk Factors and Predicting Severe COVID-19 Cases Using UK Biobank Data: Machine Learning Approach
}

Kenneth Chi-Yin Wong ${ }^{1}$, MSc; Yong Xiang ${ }^{1}$, MBBS; Liangying Yin ${ }^{1}$, MPhil; Hon-Cheong So ${ }^{1,2,3,4,5,6,7}$, MBBS, PhD

${ }^{1}$ School of Biomedical Sciences, The Chinese University of Hong Kong, Hong Kong, China

${ }^{2}$ KIZ-CUHK Joint Laboratory of Bioresources and Molecular Research of Common Diseases, Kunming Institute of Zoology and The Chinese University of Hong Kong, Kunming, China

${ }^{3}$ CUHK Shenzhen Research Institute, Shenzhen, China

${ }^{4}$ Department of Psychiatry, The Chinese University of Hong Kong, Hong Kong, China

${ }^{5}$ Margaret K.L. Cheung Research Centre for Management of Parkinsonism, The Chinese University of Hong Kong, Hong Kong, China

${ }^{6}$ Brain and Mind Institute, The Chinese University of Hong Kong, Hong Kong, China

${ }^{7}$ Hong Kong Branch of the Chinese Academy of Sciences Center for Excellence in Animal Evolution and Genetics, The Chinese University of Hong Kong, Hong Kong, China

\section{Corresponding Author:}

Hon-Cheong So, MBBS, PhD

School of Biomedical Sciences, The Chinese University of Hong Kong

RM 520A, Lo Kwee Seong Biomedical Sciences Buildiing

Chinese University of Hong Kong

Hong Kong

China

Phone: 8639439255

Email: hcso@ cuhk.edu.hk

\section{Abstract}

Background: COVID-19 is a major public health concern. Given the extent of the pandemic, it is urgent to identify risk factors associated with disease severity. More accurate prediction of those at risk of developing severe infections is of high clinical importance.

Objective: Based on the UK Biobank (UKBB), we aimed to build machine learning models to predict the risk of developing severe or fatal infections, and uncover major risk factors involved.

Methods: We first restricted the analysis to infected individuals $(\mathrm{n}=7846)$, then performed analysis at a population level, considering those with no known infection as controls (ncontrols=465,728). Hospitalization was used as a proxy for severity. A total of 97 clinical variables (collected prior to the COVID-19 outbreak) covering demographic variables, comorbidities, blood measurements (eg, hematological/liver/renal function/metabolic parameters), anthropometric measures, and other risk factors (eg, smoking/drinking) were included as predictors. We also constructed a simplified (lite) prediction model using 27 covariates that can be more easily obtained (demographic and comorbidity data). XGboost (gradient-boosted trees) was used for prediction and predictive performance was assessed by cross-validation. Variable importance was quantified by Shapley values (ShapVal), permutation importance (PermImp), and accuracy gain. Shapley dependency and interaction plots were used to evaluate the pattern of relationships between risk factors and outcomes.

Results: A total of 2386 severe and 477 fatal cases were identified. For analyses within infected individuals (n=7846), our prediction model achieved area under the receiving-operating characteristic curve (AUC-ROC) of 0.723 (95\% CI 0.711-0.736) and 0.814 (95\% CI 0.791-0.838) for severe and fatal infections, respectively. The top 5 contributing factors (sorted by ShapVal) for severity were age, number of drugs taken (cnt_tx), cystatin C (reflecting renal function), waist-to-hip ratio (WHR), and Townsend deprivation index (TDI). For mortality, the top features were age, testosterone, cnt_tx, waist circumference (WC), and red cell distribution width. For analyses involving the whole UKBB population, AUCs for severity and fatality were 0.696 (95\% CI 0.684-0.708) and 0.825 (95\% CI 0.802-0.848), respectively. The same top 5 risk factors were identified for both outcomes, namely, age, cnt_tx, WC, WHR, and TDI. Apart from the above, age, cystatin C, TDI, and cnt_tx were among the top 10 across all 4 analyses. Other diseases top ranked by ShapVal or PermImp were type 2 diabetes mellitus (T2DM), coronary artery disease, atrial fibrillation, and dementia, among others. For the "lite" models, predictive performances were broadly similar, with estimated 
AUCs of 0.716, 0.818, 0.696, and 0.830, respectively. The top ranked variables were similar to above, including age, cnt_tx, WC, sex (male), and T2DM.

Conclusions: We identified numerous baseline clinical risk factors for severe/fatal infection by XGboost. For example, age, central obesity, impaired renal function, multiple comorbidities, and cardiometabolic abnormalities may predispose to poorer outcomes. The prediction models may be useful at a population level to identify those susceptible to developing severe/fatal infections, facilitating targeted prevention strategies. A risk-prediction tool is also available online. Further replications in independent cohorts are required to verify our findings.

(JMIR Public Health Surveill 2021;7(9):e29544) doi: 10.2196/29544

\section{KEYWORDS}

prediction; COVID-19; risk factors; machine learning; pandemic; biobank; public health; prediction models; medical informatics

\section{Introduction}

COVID-19 has resulted in a pandemic affecting more than a hundred countries worldwide [1-3]. More than 177 million confirmed cases and 3.8 million fatalities have been reported worldwide as of June 19, 2021 [4], while a large number of mild or asymptomatic cases may remain undetected. Given the extent of the pandemic, it is urgent to identify risk factors that may be associated with severe disease, and to gain deeper understanding into its pathophysiology. Accurate prediction of those at risk of developing severe diseases is also clinically important.

Machine learning (ML) approaches are powerful tools to predict disease outcomes and have been increasingly applied in biomedical research. In this study we employed boosted trees (with XGboost) to predict disease outcomes and identify risk factors. This ML approach can capture complex and nonlinear interactions between variables, hence leading to better predictive power in many circumstances. In view of the COVID-19 pandemic, many ML models have been developed for diagnostic or prognostic purposes. For instance, Bayat et al [5] developed a prediction model for COVID-19 infection based on 75,991 veteran patients who were tested for the virus. The prediction was based on boosted trees and predictors included vital signs, hematology measurements, and blood biochemistries. Knight et al [6] built a model to predict in-hospital mortality for patients hospitalized with COVID-19, based on demographics, comorbidities, vital signs, and blood test results. A variety of methods including XGboost, generalized additive model, and LASSO were employed. Chung et al [7] employed deep neural networks to predict the severity of COVID-19 infection based on basic patient information, comorbidities, vital signs, clinical symptoms, and complete blood count. Wynants et al [8] performed a systematic review of COVID-19-related prediction models up to July 1, 2020, covering 169 studies describing 232 prediction models. Several recent reviews have also summarized the applications of ML methods in the study of COVID-19 (eg, [8-11]).

Here we made use of the UK Biobank (UKBB) data to build ML models to predict severity and fatality from COVID-19, and evaluated the contributing risk factors. We built prediction models not only for patients infected but also at a general population level. While predictive performance is the main concern in most previous studies, we argue that ML models can also provide important insights into individual contributing factors and the pattern of complex relationships between risk factors and the outcome. While many have studied risk factors of COVID-19 susceptibility or severity in the UKBB [12-14] or other cohorts (eg, [8,15-18]), most relied on conventional linear models. As such, nonlinear effects and interactions between variables may be missed.

We note that in the UKBB clinical data were collected years before the outbreak of infection in 2020, which may be a limitation. Ideally, the predictors should be measured at the time when the model is intended to be applied (eg, at admission). However, we believe that building ML models with previously collected clinical data is useful for reasons detailed below. First, using previously collected clinical features may facilitate the identification of potential causal risk factors. As the predictors are collected prior to the outbreak, there is no concern about reverse causality. In practice, infection itself will lead to changes in many clinical parameters (eg, glucose, inflammatory markers, liver/renal functions); hence, it is often difficult to tell the direction of effect in cross-sectional studies. We hypothesize that this study will identify general or "baseline" risk factors or laboratory measurements that may be (causally) predictive of outcome. Second, the UKBB is a huge population-based sample $(\mathrm{N}=\sim 500,000)$, and the rich clinical data collected previously enable ML models to be developed at the general population level. Importantly, there is a relative lack of such population-level ML prediction models to identify who may be at risk of developing severe COVID-19 infections. We hope this study will fill the gap, as this may have implications for prioritizing individuals for specific prevention strategies (eg, vaccination) and diagnostic testing under limited resources.

In this study we performed 4 sets of analysis. In the first 2 sets, we built ML models to predict the severity and mortality of COVID-19 among those who are tested positive for the virus. In this setting, predictive performance is of secondary concern (as predictors were not assessed at or during admission), but the predictive performance can shed light on to what extent baseline (prediagnostic) clinical characteristics contribute to severe infections. In the other 2 sets of analysis, we predicted severity and mortality of COVID-19 at the population level, considering individuals not known to be infected as "controls." Our objectives are twofold. The first is to build prediction models for severity and mortality from COVID-19. In addition, we will uncover how different risk factors and their interactions impact on disease severity. 


\section{Methods}

\section{UK Biobank Data}

The UKBB is a large-scale prospective cohort comprising nearly 500,000 individuals aged 40-69 when they were recruited in 2006-2010. Given that the first case of COVID-19 in the UK was recorded on January 31, 2020, individuals with recorded mortality before January 31, 2020 (28,931 out of 502,524 individuals) were excluded. We also excluded from subsequent analyses a very small number of individuals $(n=19)$ whose cause of mortality was COVID-19 (ICD code U07.1) but with negative test result(s) within 1 week. The current age of individuals included in our analyses ranged from 50 to 87 years, with $50.77 \%(255,170 / 502,524)$ being older than 70 . This analysis was conducted under the project number 28732. For details of the UKBB data, please also refer to Sudlow et al [19].

\section{COVID-19 Phenotypes}

COVID-19 outcome data were downloaded from data portal provided by the UKBB. Details of data release are provided in [20]. Briefly, the latest COVID test results were extracted on December 30, 2020 (last update on December 14, 2020). The data set also included an indicator on whether the patient was an inpatient when the specimen was taken. We consider inpatient (hospitalization) status as a proxy for severity, as more sophisticated indicators of severity cannot be reliably derived yet. We noted that only $10.22 \%(468,235 / 4,581,006$ infected cases, from [21] as of June 16, 2021) of patients were admitted in the UK; as such, it is likely that only the more severe cases were hospitalized. Hospitalization has also been considered as an outcome measure in many studies, including those of vaccination effectiveness [22-25], risk prediction [26,27], and genetic/clinical risk factors [28,29] underlying severe COVID-19.

In general, we required both test result and origin to be 1 (indicating positive test and inpatient origin, respectively) to qualify as an "inpatient" case. For a small number of individuals with inpatient origin $=0$ and result $=1$, but changed to origin $=1$ with result=0 within 2 weeks' time (based on the fact that median duration of viral persistence is nearly 2 weeks [30]), we still considered those as inpatient cases (ie, assume the hospitalization was related to the infection). All other patients with at least one positive SARS-CoV-2 test result were considered as "outpatient."

Data on mortality and cause of mortality were also extracted (with latest update on December 14, 2020). Individuals with recorded cause of mortality as "U07.1" were considered as having a fatal infection with laboratory-confirmed COVID-19 (please also refer to [31]). We defined a case as "severe COVID-19" if the individual is an inpatient or if the cause of mortality is U07.1.

\section{Sets of Analysis}

Four sets of analysis were performed. The first 2 sets were restricted to test-positive cases ( $\mathrm{n}=7846)$. "Severe COVID-19" $(n=2386)$ and death $(n=477)$ due to COVID-19 were treated as outcomes. Because only prediagnostic clinical data were available, the main objective of this analysis was to identify baseline risk factors for severe/fatal illness among the infected. We then performed another 2 sets of analysis with the same outcomes, but the "unaffected" group was composed of the general population $(n=465,728)$ that did not have a diagnosis of COVID-19 or were tested negative. The 4 sets of analysis were also referred to as cohorts A-D as shown in Table 1. We also constructed gender-specific prediction models.

Table 1. The four sets of analysis performed and predictive performances (full model and lite model).

\begin{tabular}{|c|c|c|c|c|c|c|c|c|}
\hline \multirow[t]{2}{*}{ Cohort } & \multirow[t]{2}{*}{ Group 1} & \multirow[t]{2}{*}{ Group 2} & \multirow[t]{2}{*}{$\mathrm{n}$ (group 1) } & \multirow[t]{2}{*}{$\mathrm{n}$ (group 2) } & \multicolumn{2}{|c|}{$\begin{array}{l}\text { Area under the } \\
\text { curve }^{\mathrm{a}}(\%)\end{array}$} & \multicolumn{2}{|c|}{$95 \%$ CI (\%) } \\
\hline & & & & & Full & Lite & Full & Lite \\
\hline A & Hospitalized or fatal cases & Nonhospitalized cases & 2386 & 5460 & 72.3 & 71.6 & 71.1-73.6 & $70.3-72.9$ \\
\hline B & Fatal cases & All other COVID-19 cases & 477 & 7369 & 81.4 & 81.8 & $79.1-83.8$ & $79.4-84.2$ \\
\hline $\mathrm{C}$ & Hospitalized or fatal cases & $\begin{array}{l}\text { UK Biobank patients without a COVID- } \\
19 \text { diagnosis or tested negative }\end{array}$ & 2386 & 465,728 & 69.6 & 69.6 & $68.4-70.8$ & $68.4-70.7$ \\
\hline $\mathrm{D}$ & Fatal cases & $\begin{array}{l}\text { UK Biobank patients without a COVID- } \\
19 \text { diagnosis or tested negative }\end{array}$ & 477 & 465,728 & 82.5 & 83.0 & $80.2-84.8$ & $80.8-85.3$ \\
\hline
\end{tabular}

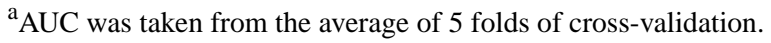

\section{Variables Included in Analysis}

We extracted a total of 97 clinical variables of potential relevance based on the literature. For details, please refer to Table S1d in Multimedia Appendix 1 and the references therein. The prediction model using all 97 variables will be referred to as the "full" model, as opposed to a simplified model ("lite" model; see below) based on mainly demographic data and medical history that can be more readily obtained. Among the 97 variables, 21 were categorical and 76 were quantitative traits.
The missing rates of variables were all below $20 \%$ (ranging from $0.0 \%$ to $19.9 \%$ for the 97 variables). We included a wide range of clinical features here, with an objective to uncover potential novel risk factors for the disease. The ML model we employed (XGboost) tends to have a low bias and high variance; however, with proper tuning of hyperparameters and regularization, overfitting can be largely avoided even when a large number of predictors are included [32]. 
The full list of variables is shown in Table S1b in Multimedia Appendix 1. Briefly, we included basic demographic variables (eg, age, sex, ethnic group, socioeconomic status as indicated by the Townsend deprivation index [TDI]), comorbidities (eg, heart diseases, type 1 and 2 diabetes mellitus [T1DM/T2DM], hypertension [HT], asthma/chronic obstructive pulmonary disease [COPD], cancer, dementia, and psychiatric disorders), indicators of general health (number of medications taken [cnt_tx], number of illnesses, etc.), blood measurements (hematology, liver and renal function measures, metabolic parameters such as lipid levels, HbA1c), anthropometric measures (eg, waist circumference [WC], waist-to-hip ratio [WHR], body mass index), and lifestyle risk factors (eg, smoking, drinking habits). Disease traits were defined based on ICD-10 diagnoses (UKBB data-field 41270), self-reported illnesses (UKBB data-field 20002), and data from follow-ups. Individuals with no records of the relevant disease from either self-reports or ICD-10 diagnoses were regarded as having no history of the disease.

\section{Imputation}

Missing values of remaining features were imputed with the $R$ package missRanger ( $R$ Foundation). The program is based on missForest [33], which is an iterative imputation approach based on random forest. It has been widely used and has been shown to produce low imputation errors and good performance in predictive models [34]. The main difference between missRanger and missForest is that the former uses the $\mathrm{R}$ package "ranger" to build random forests, which can lead to a large improvement in speed. Predictive mean matching (pmm) was also employed to avoid imputation with values not present in the original data. We employed the default parameters (pmm.k=3, num.trees=100) and default settings of ranger. Out-of-bag errors (in terms of classification errors or normalized root-mean-squared error) were computed which provides a guide to imputation accuracy.

We have also attempted to use multiple imputation by chained equation (MICE) for imputation. For our data set with nearly 500,000 individuals, MICE stopped after running for 6 hours due to memory overflow error (>64 GB), whereas missRanger finished the imputation within 3 hours successfully. We considered the computational burden of MICE as too high and therefore employed missRanger in our analyses.

Several studies have compared MissForest with MICE, and there are several advantages of missForest. For categorical variables, imputation accuracy of missForest is likely to be higher than that of MICE [35]. MissForest also runs considerably faster than MICE and is especially suitable for imputation settings where complex interactions and nonlinear relationships are likely [33]. Stekhoven et al [33] reported superior performance of missForest compared with MICE, with reduction in the proportion of falsely classified entries of up to $60 \%$. In another comparison study, missForest and MICE performed similarly but it was reported that highly correlated variables may lead to significant problems with MICE [36].

\section{XGboost Prediction Model}

XGboost with gradient-boosted trees was employed for building prediction models. Analysis was performed by the R package "xgboost." We employed a fivefold nested cross-validation strategy to develop and test the model. To avoid overoptimistic results due to choosing the best set of hyperparameters based on test performance, the test sets were not involved in hyperparameter tuning.

In each iteration, we divided the data into 5 folds, among which one-fifth was reserved for testing only. For the remaining four-fifth of the data, we further sampled four-fifth for training and one-fifth for hyperparameter tuning. The best prediction model was applied to the test set. The process was repeated 5 times. A grid-search procedure was used to search for the best combination of hyperparameters (eg, tree depth, learning rate, regularization parameters for L1/L2 penalty). The full range of hyperparameters chosen for grid search is given in Table S6 in Multimedia Appendix 1.

\section{Building a Simplified "Lite" Model}

The "full" model described above covers a wide range of predictors but some features (such as blood biochemistries) may not be readily accessible. For easier implementation in practice, we also built a simplified prediction model (also referred to as the "lite" model) based on a reduced set of 27 predictors. The reduced set of variables were chosen based on the ease of being assessed or measured, which included comorbidities (see above), anthropometric measures (BMI, weight, WC), demographic variables (eg, age, sex, ethnic group), and general indicators of health (number of medications taken, number of illnesses).

\section{Evaluating Predictive Performance and Calibration}

To evaluate the predictive performance of the prediction models, we computed the area under the receiving-operating characteristic curve (AUC-ROC), which is very widely used in clinical prediction studies. We also calculated other measures including the area under the precision-recall curve (AUC-PRC), F1 score, accuracy, and Matthews correlation coefficient (MCC). The cutoff of predicted probability for calculating the latter 3 measures was determined by optimizing the geometric mean of sensitivity and specificity.

In addition to good ability to discriminate cases from noncases, it is also important that the predicted event probabilities match with the observed probabilities (also known as calibration of a model). We assessed calibration by several measures, including the Hosmer-Lemeshow test, expected calibration error (ECE), and maximum calibration error (MCE) [37-40] across 10 equally sized bins by discretizing the predicted probabilities. We also attempted 3 approaches to further improve calibration, including Platt scaling, isotonic regression, and beta calibration [41-44]. The objective is to rescale the predicted probabilities such that they are closer to the actual probabilities of the outcome [45].

\section{Identifying and Quantifying the Effects of Important Predictors}

In this work we primarily employed Shapley value (ShapVal) $[46,47]$ to assess variable importance, which is a measure based on game theory to assess the contribution of each feature. 
ShapVal has been shown to represent a consistent and locally accurate contribution of each feature [48]. ShapVal enables local explanation of the model as it could be computed for each observation, but can also provide global importance measures. By contrast, gain and split count may produce inconsistent estimates of global importance as shown by Lundberg et al [48].

Intuitively, the ShapVal of the $i$ th feature (for individual $k$ ) is the contribution of this feature to the prediction of outcome for the individual, averaging over all possible orderings of the features (as the contribution may differ when variables enter the prediction algorithm in different orders). We ranked the global importance of features based on mean absolute ShapVal as described in previous studies [46,47]. We also attempted an alternative approach similar to "permutation importance" proposed in [49]. This method involves permuting the outcome vector to model the distribution of ShapVal under the null, and comparing the null ShapVals with the observed ShapVal. We derived a $P$ value from permutation as an alternative indicator of feature importance. A total of 500 permutations were performed for each model. To verify the validity of the permutation procedure especially under imbalanced case-control data, we also carried out a small-scale simulation study. A data set with 50,000 individuals and 10 covariates $\left(x_{1}, x_{2}, \ldots, x_{10}\right)$ were generated, where the first covariate $x_{1}$ was linearly correlated with the outcome. The control-to-case ratio was set at 976:1, same as that for cohort D. Type I error and power were assessed by repeating the entire permutation procedure for 100 randomly generated data sets (please see Multimedia Appendix 2 for details).

A related index is the Shapley interaction value [47], which computes the difference in Shapley value of feature $i$ with and without another feature $j$. ShapVal were averaged across 5 folds. Besides, we included the "gain" measure for reference, which is the reduction of loss or impurity contributed by all splits by a specific variable.

An advantage of Shapley value is that it is calculated for each individual, so how each risk factor affects a specific person's risk of infection/severity can be estimated as well. To illustrate this concept, we also produced decision plots for individuals at the highest, median, and lowest risk of each cohort.

\section{Cluster Analysis Based on Shapley Value}

We also performed cluster analysis based on ShapVal to identify subgroup of patients who share similar clinical risk factors with respect to severity of infection. As introduced in [48], this approach may be considered a form of "supervised" clustering, as the outcome (severe/fatal disease) is also taken into account in the clustering process. Unlike a traditional clustering approach based on risk factors, this approach has important advantages. First, the clusters derived may be more clinically relevant as the outcome is also considered, reducing the chance that irrelevant features contribute to the subgrouping (an irrelevant feature will have relatively small variations in ShapVal and will not contribute substantially to clustering). Second, this approach essentially considers all features on the same "scale," as ShapVal is computed with respect to the outcome. Input features are often of different units and scales, but ShapVal considers feature contributions to the outcome as the unit of measure. Because of computational cost concerns, here we only performed clustering on cohorts A (nonsevere vs severe infection) and B (fatal vs nonfatal infection).

\section{K-Means Sparse Clustering}

Here we performed k-means sparse clustering to uncover underlying patient subgroups based on ShapVal of risk factors. As the number of features included is large but not all may contribute to the underlying subgroups, we employed sparse clustering which incorporates feature selection in the clustering process. The R package "sparcl" was employed. To perform sparse k-means clustering, we need to predetermine the number of clusters and tuning parameter (L1 penalty) for feature selection [50]. The optimal number of clusters was assumed to be the same as that in k-means clustering, which was determined by the silhouette index. The tuning parameter (L1 bound) was set to range between 2 and 6 with an interval of 0.4 . Then the gap statistic [51] was used to determine the optimal tuning parameter.

\section{Results}

An overview of the sample sizes in each set of analysis is presented in Table 1. Please also refer to Table S1a and S1b in Multimedia Appendix 1 for a detailed summary of case counts and covariates.

\section{Simulation Results for the Permutation Testing Approach}

Simulation results for the validity of permutation $P$ values are presented in Table S8 in Multimedia Appendix 1. We observed no inflation of type I error (false-positive rate) despite the imbalanced case-to-control ratio. At a $P$ value threshold of 0.05 , the proportion of results with $P<.05$ for $x_{2}$ to $x_{10}$ (variables with null effect) remained less than 0.05 for different effect sizes of the predictor (please also refer to Multimedia Appendix 2).

\section{Prediction Performance of the XGboost Model for Risk and Severity of Infection}

\section{$A U C-R O C$ and Other Results}

We performed 5-fold cross-validation and the average AUC under the ROC curve is given in Table 1 and Table S2a in Multimedia Appendix 1. Here we describe the results for the full models first. We observed better predictive performances in cohorts B (fatal cases vs outpatient cases) and D (fatal cases vs population with no known infection), where fatalities from COVID-19 were modeled. The corresponding mean AUC-ROC values were 0.814 (95\% CI 0.791-0.838) and 0.825 (95\% CI $0.802-0.848)$, respectively. The mean AUC-ROC for cohort A (hospitalized/fatal cases vs other cases) was 0.723 (95\% CI 0.711-0.736) and that for cohort $\mathrm{C}$ (hospitalized/fatal cases vs population with no known infection) was 0.696 (95\% CI 0.684-0.708).

As for the "lite" models which included a reduced set of predictors, the predictive performances in terms of AUC are broadly similar, with estimated AUC-ROC for cohorts A-D of $0.716,0.818,0.696$, and 0.830 , respectively. 
The results of other predictive indices are listed in Table S2b in Multimedia Appendix 1. Estimates of AUC-PRC were the highest for cohorts $\mathrm{A}$ and $\mathrm{B}(0.535$ and 0.171 , respectively $)$ and much lower for cohorts $\mathrm{C}$ and $\mathrm{D}$ ( 0.007 and 0.006 , respectively). This is expected due to the much higher prevalence of outcome in the first 2 cohorts. AUC-PRC may be approximated by the average precision (please refer to [52] for further details).

We also conducted sex-stratified analysis (Table S2a in Multimedia Appendix 1). The resulting AUC-ROC was similar to the overall analysis in males (except for cohort D), but generally lower in females. This may be partially explained by lower number of severe and fatal cases in females, which leads to greater difficulty in model training.

Proportion of Cases Explained by Individuals at the Top $k \%$ of Predicted Risk

We also computed the proportion of cases explained by individuals at the highest $k \%$ of predicted risks (Table 2). For example, considering the full model, for prediction of mortality among infected individuals (cohort B), individuals at the highest
$5 \%, 10 \%$, and $20 \%$ of predicted risks explain $17.4 \%$ (83/477), $32.7 \%(156 / 477)$, and $52.0 \%$ (248/477) of total fatalities, respectively. As for prediction in the population (cohort D), individuals at the highest $5 \%, 10 \%$, and $20 \%$ of predicted risks explain $32.5 \%$ (155/477), $45.7 \%$ (218/477), and $63.5 \%$ (303/477) of total fatalities, respectively. For prediction of severe disease among the infected (cohort A), individuals at the highest $5 \%, 10 \%$, and $20 \%$ of predicted risks explain $11.2 \%$ (267/2386), $21.6 \%(515 / 2386)$, and $38.2 \%(911 / 2386)$ of total cases, respectively, while more than half $(1272 / 2386,53.3 \%)$ of cases are explained by people at the top $30 \%$ of predicted risks. For prediction of severe cases in the population (cohort C), the corresponding figures were $19.7 \%$ (470/2386), $29.3 \%$ (700/2386), and 42.7\% (1019/2386), respectively, and more than half $(1260 / 2386,52.8 \%)$ of cases are explained by people at the top $30 \%$ of predicted risks. Similar figures were observed for full and lite models in general.

These results showed in general a strong enrichment of cases among those predicted to have high risks, indicating good model discriminatory ability. 
Table 2. Relative risk (RR) comparing subjects in the top and bottom k\% of predicted risks and proportion of cases explained by those at top k\% of predicted risk.

\begin{tabular}{|c|c|c|c|c|c|c|c|c|}
\hline \multicolumn{5}{|c|}{ Full model } & \multicolumn{4}{|l|}{ Lite model } \\
\hline$k$ & $\begin{array}{l}\text { Risk in top } \\
\mathrm{k} \%{ }^{\mathrm{a}, \mathrm{b}}\end{array}$ & $\begin{array}{l}\text { Risk in bottom } \\
\mathrm{k} \%\end{array}$ & $\mathrm{RR}$ & $\begin{array}{l}\text { Proportion of cases } \\
\text { explained by top } \mathrm{k} \%\end{array}$ & Risk in top $\mathrm{k} \%^{\mathrm{a}, \mathrm{b}}$ & Risk in bottom $\mathrm{k} \%$ & $\mathrm{RR}$ & $\begin{array}{l}\text { Proportion of } \\
\text { cases explained } \\
\text { by top k\% }\end{array}$ \\
\hline
\end{tabular}

\section{Cohort A}

\subsection{8}

4.56

0.112

0.691

0.158

$4.37 \quad 0.113$

$10 \quad 0.654$

$4.74 \quad 0.216$

0.644

0.157

$4.10 \quad 0.211$

$20 \quad 0.579$

0.145

$4.00 \quad 0.382$

0.581

0.153

$3.79 \quad 0.381$

$30 \quad 0.540$

0.148

3.65

0.533

0.533

0.152

$3.50 \quad 0.526$

$40 \quad 0.489$

0.152

3.20

0.479

$50 \quad 0.443$

0.166

$2.67 \quad 0.730$

0.439

0.158

$3.03 \quad 0.630$

0.170

$2.59 \quad 0.720$

Cohort B

$\begin{array}{lllll}5 & 0.214 & 0.000 & \text { Infinity } & 0.174 \\ 10 & 0.200 & 0.001 & 158.20 & 0.327 \\ 20 & 0.171 & 0.008 & 22.42 & 0.562 \\ 30 & 0.148 & 0.009 & 16.57 & 0.727 \\ 40 & 0.127 & 0.009 & 14.21 & 0.830 \\ 50 & 0.111 & 0.010 & 10.94 & 0.916\end{array}$

0.212
0.216
0.188
0.155
0.131
0.111

0.003

$84.27 \quad 0.174$

0.008

$28.38 \quad 0.352$

0.008

$24.59 \quad 0.618$

0.008

$19.21 \quad 0.763$

Cohort C

$\begin{array}{lllll}5 & 0.0201 & 0.0017 & 11.76 & 0.197 \\ 10 & 0.0149 & 0.0021 & 6.98 & 0.293 \\ 20 & 0.0109 & 0.0023 & 4.67 & 0.427 \\ 30 & 0.0090 & 0.0030 & 2.99 & 0.528 \\ 40 & 0.0075 & 0.0033 & 2.27 & 0.590 \\ 50 & 0.0069 & 0.0033 & 2.09 & 0.678\end{array}$

$\begin{array}{llll}0.0210 & 0.0013 & 15.88 & 0.207 \\ 0.0158 & 0.0012 & 12.95 & 0.310 \\ 0.0118 & 0.0021 & 5.71 & 0.462 \\ 0.0097 & 0.0027 & 3.57 & 0.573 \\ 0.0084 & 0.0026 & 3.20 & 0.656 \\ 0.0074 & 0.0028 & 2.63 & 0.725\end{array}$

Cohort D

\begin{tabular}{llllllllll}
5 & 0.0067 & 0.00000 & Infinity & 0.325 & 0.0068 & 0.00000 & $\begin{array}{l}\text { Infini- } \\
\text { ty }\end{array}$ & 0.333 & \\
10 & 0.0047 & 0.00002 & 218.02 & 0.457 & 0.0047 & 0.00006 & 73.67 & 0.463 \\
20 & 0.0033 & 0.00011 & 30.30 & 0.635 & 0.0032 & 0.00009 & 36.75 & 0.616 \\
30 & 0.0026 & 0.00014 & 18.74 & 0.746 & 0.0027 & 0.00011 & 23.38 & 0.784 \\
40 & 0.0021 & 0.00016 & 13.17 & 0.828 & 0.0022 & 0.00013 & 16.68 & 0.874 \\
50 & 0.0018 & 0.00022 & 8.35 & 0.893 & 0.0019 & 0.00015 & 13.03 & 0.929 \\
\hline
\end{tabular}

a'Top $\mathrm{k} \%$ ' refers to top $\mathrm{k} \%$ of predicted probability of outcome by XGboost.

$\mathrm{b}$ 'Risk in top $\mathrm{k} \%$ ' refers to the actual probability of the outcome (severe disease or fatality) within the patients belonging to the highest $\mathrm{k} \%$ of predicted risks.

\section{Relative Risk of Actual Outcome Probabilities, Comparing Those at the Highest and Lowest $k \%$ of Predicted Risks}

We also computed the relative risk (RR) of infection or severe disease by comparing individuals at the highest and lowest $k \%$ of predicted risks (Table 2). For example, considering the full model, if we compare the actual probability of outcome at the top decile (top 10\%) against those at the bottom decile of predicted risks, the RR was 4.74, 158.2, 6.98, and 218.02, respectively, for cohorts A to D. If we compare the top $20 \%$ against the lowest $20 \%$ of predicted risks, the corresponding RRs were 4.00, 22.42, 4.67, and 30.30. The RRs for the lite model were similar for cohorts $\mathrm{A}$ and $\mathrm{C}$, but were smaller for cohorts $\mathrm{B}$ and $\mathrm{D}$ when the comparison was made at the more extreme ends of predicted risks.

We observed large RRs for cohorts B and D, suggesting that the prediction models were able to discriminate individuals at the highest and lowest risks of fatality very well. RRs for cohorts 
B and D were much larger than those for cohorts A and C, indicating that the model predicted fatality better than severe disease.

\section{Calibration}

As for calibration, please refer to Figures S6 and S7 in Multimedia Appendix 3. For full models, cohort A was well-calibrated (without using other methods for recalibration) with ECE of 0.022 and MCE of 0.044 only. For other models, the ECE and MCE were generally larger, probably due to large difficulty in calibration with a much lower probability of the outcome. The best ECEs (after recalibration by 1 of the 3 methods) were $0.11,0.14$, and 0.02 , respectively, for cohorts B-D. The Hosmer-Lemeshow test was nonsignificant in cohorts $\mathrm{C}$ and D ( $P=.99$ and .98 , respectively). For the "lite" models, the best ECEs were 0.017, 0.043, 0.024, and 0.089, respectively, for cohorts A-D, with nonsignificant Hosmer-Lemeshow test results except for cohort B (Hosmer-Lemeshow $P=.49$, .003, .97 , and .41 for cohorts A-D, respectively).

\section{Results From Cluster Analysis Based on ShapVal}

Figures 1 and S11 in Multimedia Appendix 3 show the results based on sparse k-means clustering. We performed clustering separately in cases and controls to uncover patient subgroups with different clinical background. Here we focus on clustering results within cases. As the number of variables is large, we only showed the variables that were statistically significant $(P<.05$ from $t$ test or ANOVA) across the clusters in the figures.
For cohort A, we found 2 clusters as the optimal solution. The first cluster has higher ShapVal for most risk factors, especially age, but also cnt_tx, HbA1c, cystatin C, high-density lipoprotein (HDL)-cholesterol, and HT. ShapVal for WHR was positive for the first group but negative for the second group. The first cluster may represent a subgroup of severe cases with a larger number of clinical risk factors/comorbidities and advanced age, while the second cluster may be a distinct group with less conventional risk factors (especially obesity), yet is susceptible to severe infections perhaps due to other (unmeasured) factors, such as genetics.

Considering cohort B cases (fatal infections), the optimal solution comprised 3 clusters. Interestingly, the first and third clusters seemed to be markedly different with respect to their risk factor profiles. Mean ShapVal for age was largely negative for the first cluster but highly positive for the other 2 clusters. By contrast, mean ShapVal for WC was markedly higher and positive for the first cluster. The third cluster was characterized by the highest mean ShapVal for age, and higher (positive) ShapVal for mainly cnt_tx, HbA1c, and T2DM. The results suggest that there may exist pathophysiologically distinct subgroups of patients with fatal infection. The first cluster represents a subgroup with younger age but with higher proportion of obesity. The third cluster represents another subgroup with advanced age, more comorbidities, and higher proportion of glucose abnormalities or T2DM. The second cluster is in between. 
Figure 1. Results of sparse k-means clustering based on Shapley values (ShapVal) in cohorts A (hospitalized cases) and B (fatal cases). The y-axis indicates the ShapVal and only those risk Factors with significant differences $(P<.05$ in t-test or ANOVA) across clusters were shown on the $\mathrm{x}$-axis. AF: atrial fibrillation; CAD: coronary artery disease; Hb: hemoglobin; HbA1c: hemoglobin A1c; HDL: high-density lipoprotein; LDL: low-density lipoprotein; RBC: red blood cell; RF: risk factor; SHBG: sex hormone binding globulin; T1DM: type 1 diabetes; T2DM: type 2 diabetes.

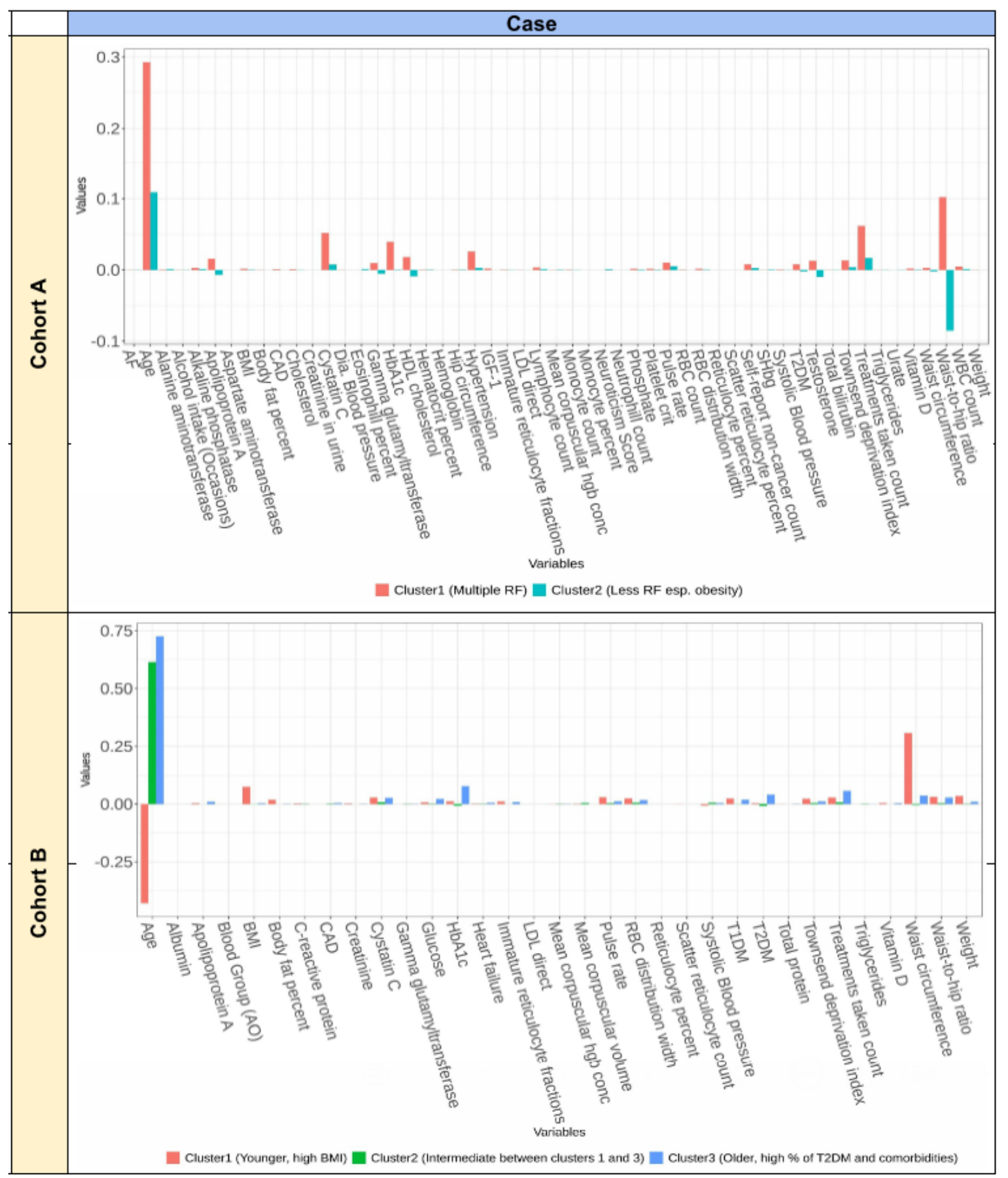

\section{Important Contributing Variables Identified}

\section{Overview}

Here we primarily report the results of the full model as a more complete set of predictors is included. The Shapley dependence plots (ranked by mean absolute ShapVal) of the top 15 features (full model) are shown in Figure 2 and those of the top 6 features for the lite model are presented in Figure 3. For more complete plots (up to 30 variables) with ranking by mean abs(ShapVal) or permutation $P$ values, please refer to Figures $\mathrm{S} 1-\mathrm{S} 4$ in Multimedia Appendix 3.

Full ShapVal analysis results on all variables are given in Tables S3a-c in Multimedia Appendix 1. The top 10 variables (ranked by either ShapVal or permutation $P$ value) from the full model 
are presented in Tables 3 and 4 while the top 5 from the lite model are presented in Tables 5 and 6 . We also included variable importance by gain, and plots are presented in Figure S5a and S5b in Multimedia Appendix 3.

Figure 2. Shapley value dependence plots of the top 15 risk factors ranked by mean abs(shapley value) (full model) for cohorts A, B, C, and D, respectively. Shapley value (y-axis) is computed on a log-odds scale. Every unit increase of ShapVal corresponds to an odds ratio (OR) of exp $(1)=2.72$ compared with the baseline. Positive ShapVal indicates increase in the odds of the outcome and vice versa. CAD: coronary artery disease; COPD: chronic obstructive pulmonary disease; HDL: high-density lipoprotein; RBC: red blood cell; T2DM: type 2 diabetes mellitus.

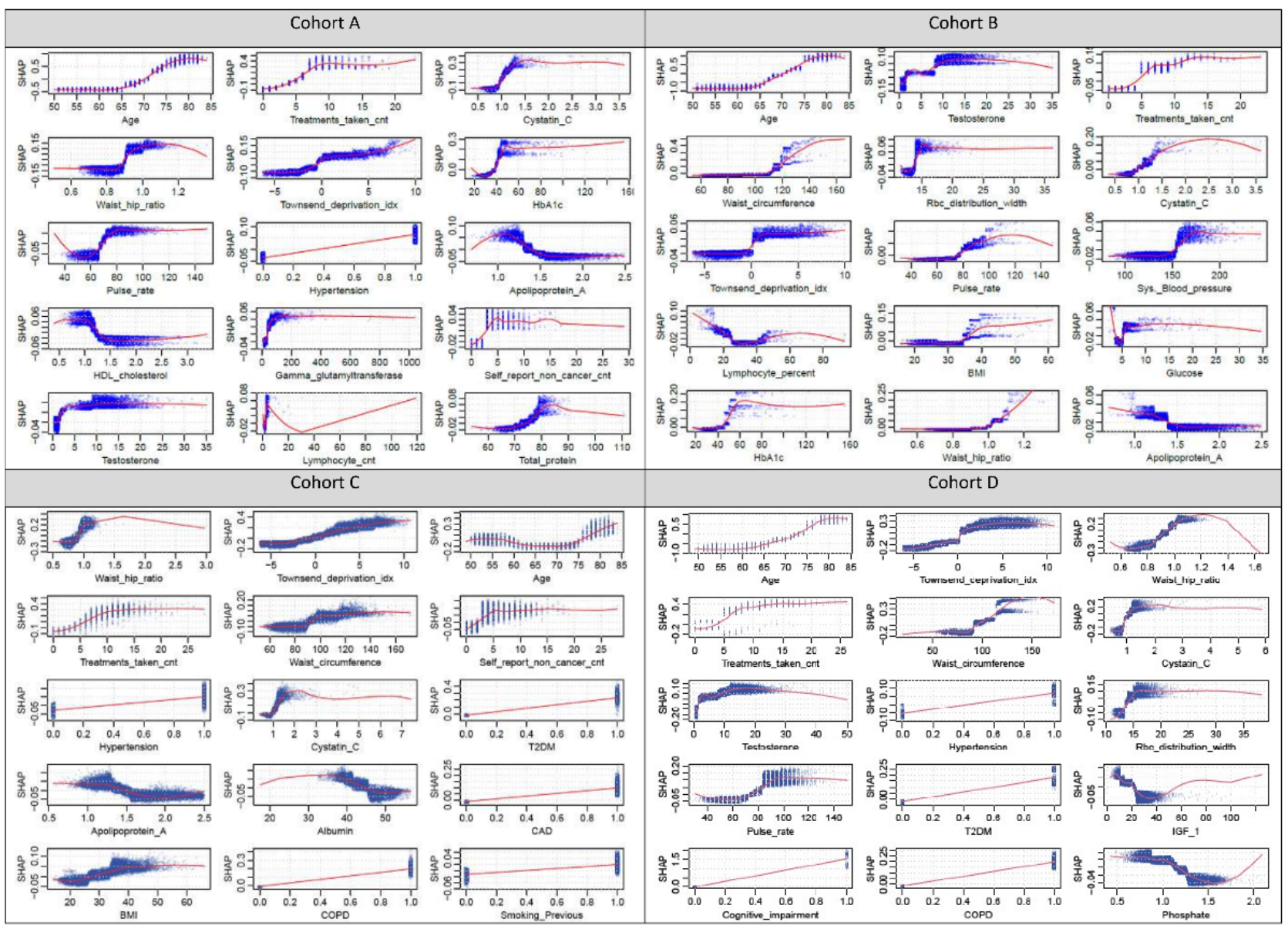

Figure 3. ShapVal dependence plots of the top 6 risk factors ranked by mean abs(shapley value) (lite model) for cohorts A, B, C, and D, respectively. T2DM: type 2 diabetes mellitus.

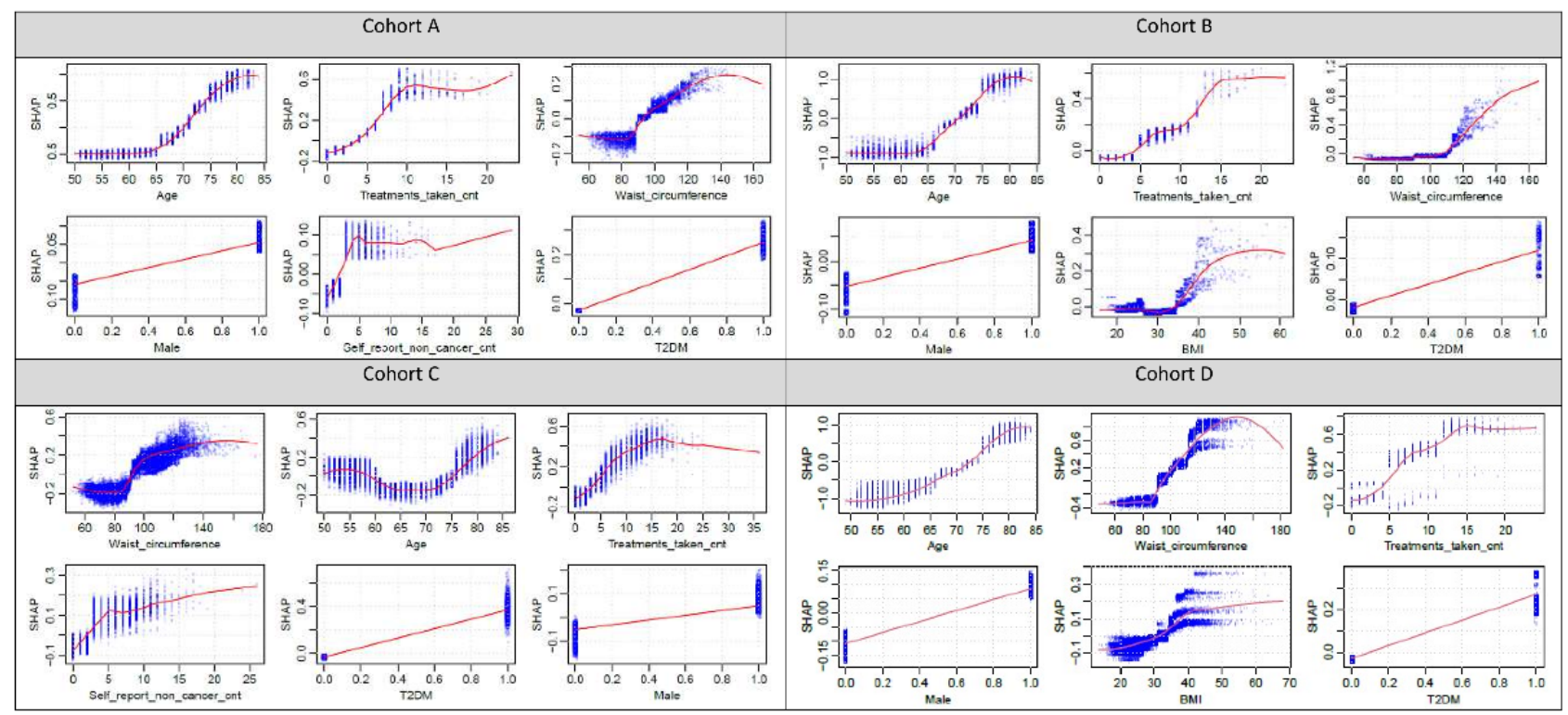


Table 3. Top 10 risk factors ranked by mean absolute Shapley value for cohorts A, B, C, and D (full model).

\begin{tabular}{|c|c|c|}
\hline Risk factor & ShapVal & $P$ value \\
\hline \multicolumn{3}{|l|}{ Cohort A } \\
\hline Age & 0.442 & .002 \\
\hline Treatments taken count & 0.093 & .002 \\
\hline Cystatin C & 0.088 & .002 \\
\hline Waist-to-hip ratio & 0.085 & .002 \\
\hline Townsend deprivation index & 0.059 & .004 \\
\hline $\mathrm{HbA} 1 \mathrm{c}^{\mathrm{a}}$ & 0.056 & .002 \\
\hline Pulse rate & 0.048 & .002 \\
\hline Hypertension & 0.048 & .002 \\
\hline Apolipoprotein A & 0.027 & .016 \\
\hline HDL $^{\mathrm{b}}$ cholesterol & 0.026 & .016 \\
\hline \multicolumn{3}{|l|}{ Cohort B } \\
\hline Age & 0.708 & .002 \\
\hline Testosterone & 0.069 & .002 \\
\hline Treatments taken count & 0.048 & .002 \\
\hline Waist circumference & 0.035 & .002 \\
\hline $\mathrm{RBC}^{\mathrm{c}}$ distribution width & 0.027 & .002 \\
\hline Cystatin C & 0.024 & .002 \\
\hline Townsend deprivation index & 0.023 & .002 \\
\hline Pulse rate & 0.019 & .004 \\
\hline Systolic blood pressure & 0.016 & .002 \\
\hline Lymphocyte percentage & 0.015 & .004 \\
\hline \multicolumn{3}{|l|}{ Cohort C } \\
\hline Waist-to-hip ratio & 0.113 & .002 \\
\hline Townsend deprivation index & 0.096 & .002 \\
\hline Age & 0.088 & .002 \\
\hline Treatments taken count & 0.063 & .002 \\
\hline Waist circumference & 0.044 & .002 \\
\hline Self-report: noncancer count & 0.043 & .002 \\
\hline Hypertension & 0.036 & .002 \\
\hline Cystatin C & 0.030 & .024 \\
\hline $\mathrm{T} 2 \mathrm{DM}$ & 0.030 & .002 \\
\hline Apolipoprotein A & 0.024 & .052 \\
\hline \multicolumn{3}{|l|}{ Cohort D } \\
\hline Age & 0.519 & .002 \\
\hline Townsend deprivation index & 0.136 & .002 \\
\hline Waist-to-hip ratio & 0.131 & .002 \\
\hline Treatments taken count & 0.115 & .002 \\
\hline Waist circumference & 0.110 & .002 \\
\hline Cystatin C & 0.096 & .002 \\
\hline Testosterone & 0.086 & .002 \\
\hline
\end{tabular}




\begin{tabular}{lrr}
\hline Risk factor & ShapVal & $P$ value \\
\hline Hypertension & 0.061 & .002 \\
RBC distribution width & 0.046 & .002 \\
Pulse rate & 0.036 & .006 \\
\hline
\end{tabular}

${ }^{\mathrm{a}} \mathrm{HbA1c}$ : hemoglobin A1c.

${ }^{\mathrm{b}} \mathrm{HDL}$ : high-density lipoprotein.

${ }^{c}$ RBC: red blood cell.

${ }^{\mathrm{d}}$ T2DM: type 2 diabetes mellitus. 
Table 4. Top 10 risk factors ranked by P-value, listing only factors which are not yet included in for cohorts A, B, C, and D (full model).

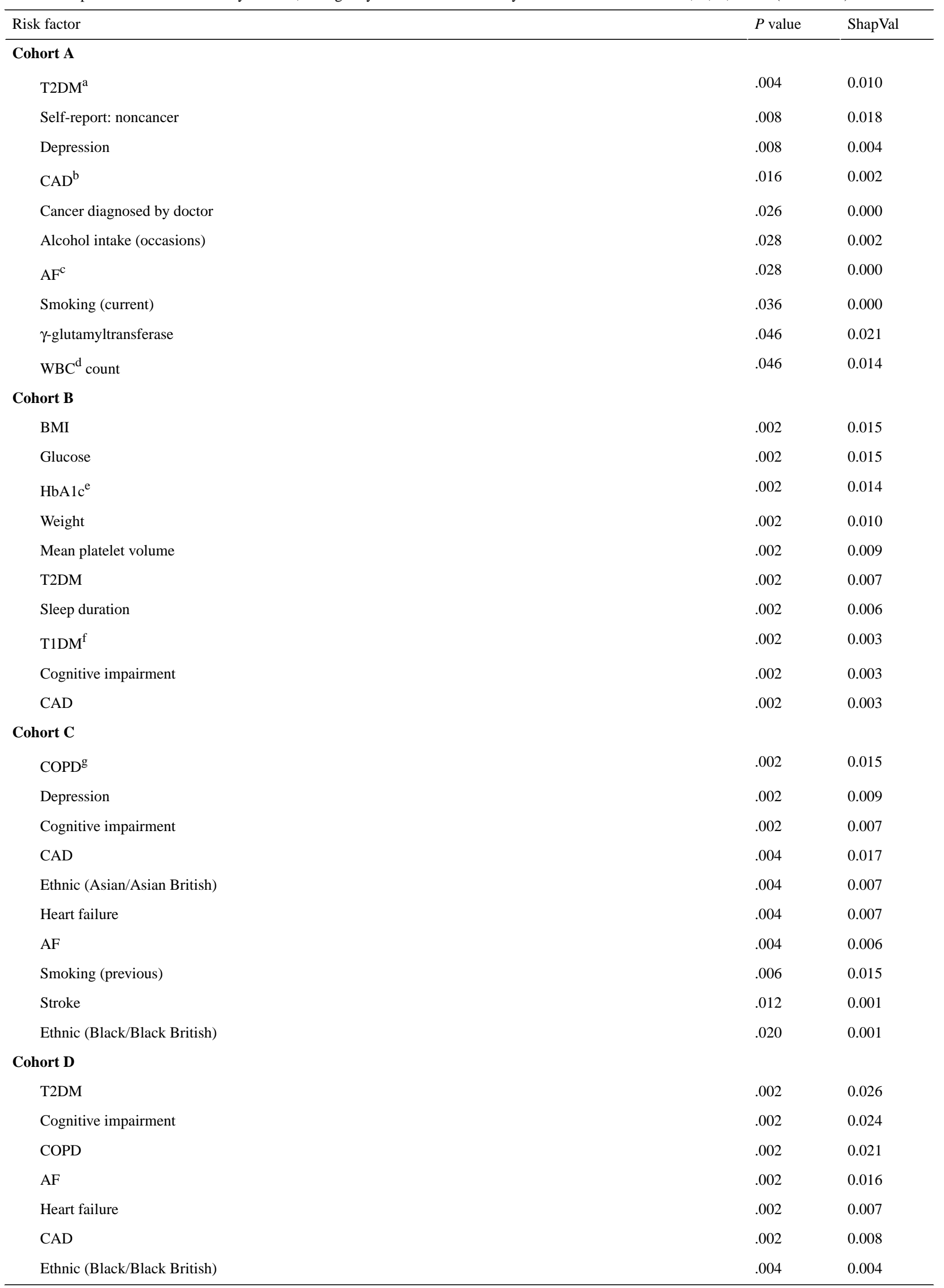




\begin{tabular}{lcc}
\hline Risk factor & $P$ value & ShapVal \\
\hline Stroke & .004 & 0.002 \\
Alcohol drinker (current) & .004 & 0.001 \\
Smoking (previous) & .006 & 0.003 \\
\hline
\end{tabular}

${ }^{\mathrm{a}}$ T2DM: type 2 diabetes mellitus.

${ }^{\mathrm{b}} \mathrm{CAD}$ : coronary artery disease.

${ }^{\mathrm{c}} \mathrm{AF}$ : atrial fibrillation.

${ }^{\mathrm{d}} \mathrm{WBC}$ : white blood cell.

${ }^{\mathrm{e}} \mathrm{HbA1c}$ : hemoglobin A1c

T1DM: type 1 diabetes mellitus.

${ }^{\mathrm{g}}$ COPD: chronic obstructive pulmonary disease.

Table 5. Top 5 risk factors ranked by mean absolute Shapley value for cohorts A, B, C, and D (lite model).

\begin{tabular}{|c|c|c|}
\hline Risk factor & ShapVal & $P$ value \\
\hline \multicolumn{3}{|l|}{ Cohort A } \\
\hline Age & 0.496 & .002 \\
\hline Treatments taken count & 0.121 & .002 \\
\hline Waist circumference & 0.085 & .002 \\
\hline Male & 0.058 & .002 \\
\hline Self-report: noncancer count & 0.054 & .004 \\
\hline \multicolumn{3}{|l|}{ Cohort B } \\
\hline Age & 0.721 & .002 \\
\hline Treatments taken count & 0.079 & .014 \\
\hline Waist circumference & 0.071 & .040 \\
\hline Male & 0.048 & .010 \\
\hline BMI & 0.034 & .242 \\
\hline \multicolumn{3}{|l|}{ Cohort C } \\
\hline Waist circumference & 0.153 & .002 \\
\hline Age & 0.120 & .002 \\
\hline Treatments taken count & 0.102 & .002 \\
\hline Self-report: noncancer count & 0.064 & .002 \\
\hline $\mathrm{T}_{2} \mathrm{DM}^{\mathrm{a}}$ & 0.050 & .002 \\
\hline \multicolumn{3}{|l|}{ Cohort D } \\
\hline Age & 0.056 & .002 \\
\hline Waist circumference & 0.248 & .002 \\
\hline Treatments taken count & 0.154 & .002 \\
\hline Male & 0.098 & .002 \\
\hline BMI & 0.043 & .036 \\
\hline
\end{tabular}

aT2DM: type 2 diabetes mellitus. 
Table 6. Top 5 risk factors ranked by $P$ value, listing only factors which are not yet included in for cohorts A, B, C, and D (lite model).

\begin{tabular}{|c|c|c|}
\hline Risk factor & $P$ value & ShapVal \\
\hline \multicolumn{3}{|l|}{ Cohort A } \\
\hline $\mathrm{T} 2 \mathrm{DM}^{\mathrm{a}}$ & .002 & 0.047 \\
\hline Smoking (current) & .004 & 0.026 \\
\hline Depression & .016 & 0.015 \\
\hline Alcohol drinker (current) & .020 & 0.013 \\
\hline $\mathrm{CAD}^{\mathrm{b}}$ & .022 & 0.010 \\
\hline \multicolumn{3}{|l|}{ Cohort B } \\
\hline $\mathrm{T} 2 \mathrm{DM}$ & .006 & 0.027 \\
\hline Cognitive impairment & .006 & 0.015 \\
\hline $\mathrm{T}^{\mathrm{DDM}} \mathrm{D}^{\mathrm{c}}$ & .020 & 0.009 \\
\hline Bipolar & .024 & 0.006 \\
\hline $\mathrm{AF}^{\mathrm{d}}$ & .036 & 0.011 \\
\hline \multicolumn{3}{|l|}{ Cohort C } \\
\hline $\mathrm{COPD}^{\mathrm{e}}$ & .002 & 0.024 \\
\hline Ethnic (Asian/British Asian) & .002 & 0.016 \\
\hline Cognitive impairment & .002 & 0.008 \\
\hline Male & .004 & 0.049 \\
\hline CAD & .004 & 0.023 \\
\hline \multicolumn{3}{|l|}{ Cohort D } \\
\hline $\mathrm{T} 2 \mathrm{DM}$ & .002 & 0.043 \\
\hline COPD & .002 & 0.039 \\
\hline Cognitive impairment & .002 & 0.029 \\
\hline $\mathrm{AF}$ & .002 & 0.024 \\
\hline Ethnic (Black/Black British) & .002 & 0.016 \\
\hline
\end{tabular}

T2DM: type 2 diabetes mellitus.

${ }^{\mathrm{b}} \mathrm{CAD}$ : coronary artery disease.

${ }^{\mathrm{c}}$ T1DM: type 1 diabetes mellitus.

${ }^{\mathrm{d}} \mathrm{AF}$ : atrial fibrillation.

${ }^{\mathrm{e}} \mathrm{COPD}$ : chronic obstructive pulmonary disease.

As for interaction analyses, top results are presented in Table 7 and full results in Tables S4 and S5 in Multimedia Appendix 1. Plots are presented in Figure 4 (top 2 interacting pairs from

Note that ShapVal is measured on the log-odds scale. Every unit increase of ShapVal corresponds to an odds ratio of $\exp (1)=2.72$. Positive ShapVal indicates increase in the odds each model) and Figures S8 and S9 in Multimedia Appendix 3 of outcome and vice versa. (top 6 interacting pairs) 
Table 7. Top interacting pairs of variables ranked by ShapVal (full model).

\begin{tabular}{|c|c|c|}
\hline Risk factor 1 & Risk factor 2 & Value \\
\hline \multicolumn{3}{|l|}{ Cohort A } \\
\hline Waist-to-hip ratio & Age & 150 \\
\hline Treatments taken count & Age & 149 \\
\hline HDL $^{\mathrm{a}}$ cholesterol & Age & 86 \\
\hline Age & Hypertension & 85 \\
\hline Cystatin C & Age & 84 \\
\hline \multicolumn{3}{|l|}{ Cohort B } \\
\hline Testosterone & Age & 195 \\
\hline Waist circumference & Age & 95 \\
\hline BMI & Age & 82 \\
\hline Treatments taken count & Age & 63 \\
\hline Pulse rate & Age & 57 \\
\hline \multicolumn{3}{|l|}{ Cohort C } \\
\hline Waist-to-hip ratio & Age & 709 \\
\hline Waist-to-hip ratio & Treatments taken count & 494 \\
\hline Townsend deprivation index & Treatments taken count & 481 \\
\hline Townsend deprivation index & Waist-to-hip ratio & 450 \\
\hline Albumin & Waist-to-hip ratio & 407 \\
\hline \multicolumn{3}{|l|}{ Cohort D } \\
\hline Waist circumference & Age & 859 \\
\hline Testosterone & Age & 780 \\
\hline Townsend deprivation index & Age & 725 \\
\hline Waist-to-hip ratio & Age & 603 \\
\hline Age & Hypertension & 585 \\
\hline
\end{tabular}

${ }^{\mathrm{a} H D L}$ : high-density lipoprotein. 
Figure 4. ShapVal interaction plots of the full model for the top 4 interacting pairs of cohorts A, B, C, and D, respectively.

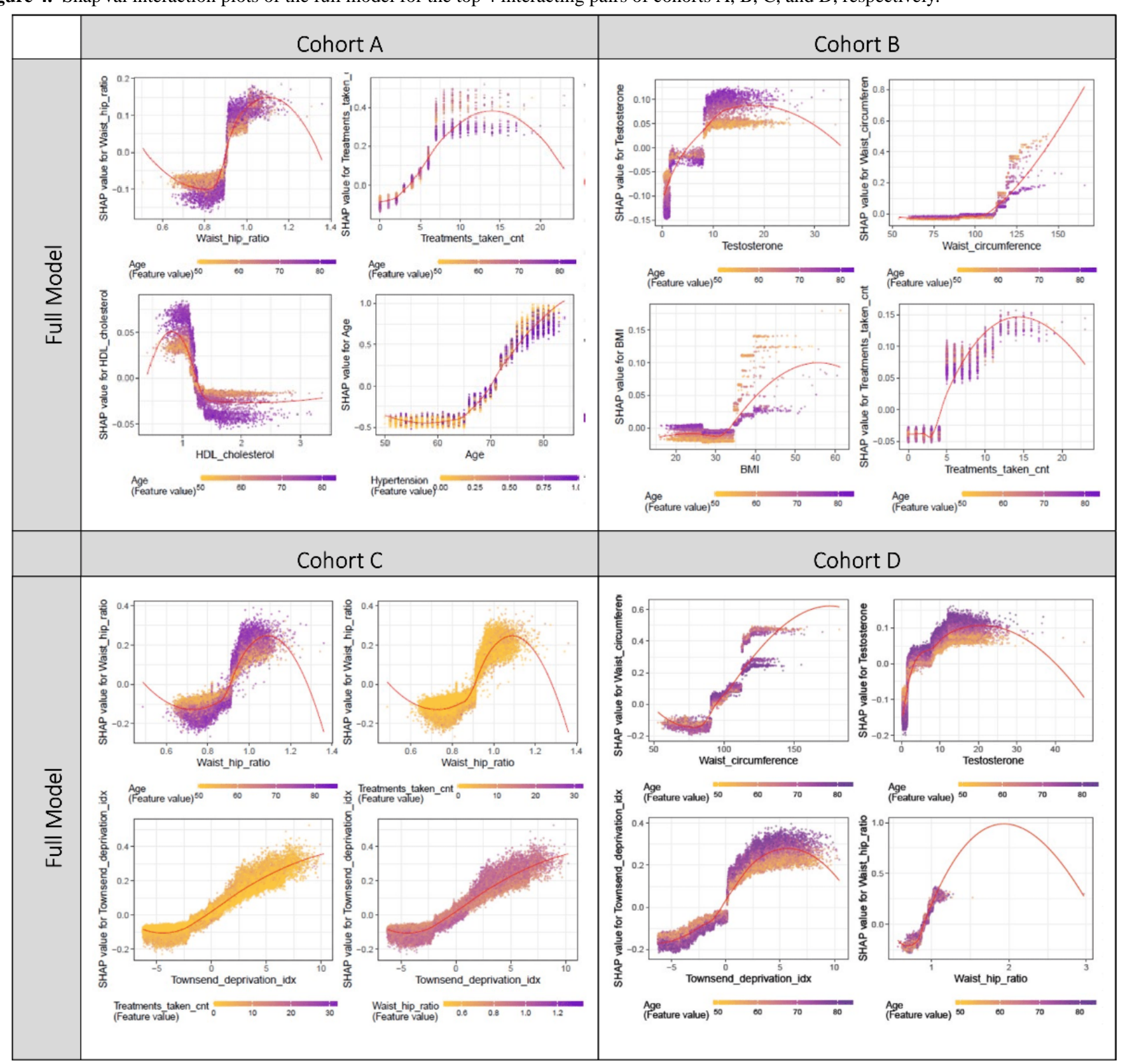

\section{Cohort A (Hospitalized/Fatal Cases vs Outpatient Cases)}

The top 5 contributing features by ShapVal included age, number of medications received (cnt_tx), cystatin C, TDI, and WHR, followed by HbA1c. Higher levels of these risk factors generally lead to higher disease severity among the infected. Interestingly, Shapley dependence plots revealed potential nonlinear and "threshold" effects of risk factors on the outcome. For example, age of 65 or above was associated with a markedly increased risk of severe/fatal infection. Markedly elevated risks were also observed for $\mathrm{HbA1c}$ levels over $40 \mathrm{mmol} / \mathrm{mol}$ and 5 or more drugs received. Impaired renal function (IRF; raised cystatin $\mathrm{C}>1 \mathrm{mg} / \mathrm{L}$ ) was also linked to worse outcomes. For WHR, levels of 0.9 or higher appeared to be associated with a marked increase in risks. For other features, please refer to Figure 2. We note that at the extreme ends of variables, the observations are often sparse, so the trend shown by the Loess curve may not be reliable (this also applies to other cohorts). Variable importance based on gain revealed similar patterns of important features (Figures S5a and S5b in Multimedia Appendix 3).

If we consider the " $P$ value" or permutation importance (PermImp) measure, variables with top 10 (absolute) ShapVal also showed significant $P$ values ( $P<.05$ for all cases). T2DM was among the top 10 by PermImp but not by ShapVal. Depression and coronary artery disease (CAD) also showed low $P$ values $(P<.02)$, but were not listed among the top 30 by ShapVal.

Regarding interactions between variables, most of the top interacting pairs involved age (Figure 4 and Tables S4 and S5 in Multimedia Appendix 1). For example, younger individuals were observed to have more extreme ShapVal at similar ranges of cnt_tx. The effect of WHR on severity was more marked among the elderly, and the same was true for HDL-cholesterol (low HDL is a risk factor). 


\section{Model B (Fatal Cases vs Outpatient Cases)}

The top 5 contributing variables by ShapVal included age, testosterone (which may reflect the effect of gender), cnt_tx, WC, and red blood cell distribution width (RDW), which were followed by cystatin C, TDI, pulse rate, systolic blood pressure (SBP), and percentage of lymphocytes. Again, certain nonlinear and "threshold" effects appeared to be present for many top-ranked features. For age, the risk for mortality was more marked beyond 65 years. Higher levels of all the above risk factors (RFs) (except percentage of lymphocytes, which showed a U-shaped relationship) were associated with higher mortality, but the effects were nonlinear. Regarding the top results based on PermImp, 8 out of 10 predictors ranked high by ShapVal also had the lowest $P$ values (lowest $P$ value of .002 since we performed 500 permutations). Other top-ranked features $(P=.002)$ included HbA1c, type 1 and T2DM, weight, mean platelet volume, etc.

Variable importance based on gain yielded similar results (Figures S5a and S5b in Multimedia Appendix 3). As for interactions between the variables, again interactions were most prominent with age (Figure 4). For example, the effects of WC and BMI (when exceeding a threshold of around $110 \mathrm{~cm}$ and $35 \mathrm{~kg} / \mathrm{m}^{2}$, respectively) on mortality were more prominent among younger individuals. The effects of testosterone and $\mathrm{HbA1c}$, however, were more marked in older individuals.

\section{Model C (Hospitalized/Fatal Cases vs Population With No Known Infection)}

Based on ShapVal, WHR was the top contributing variable and WC was ranked fifth, suggesting that central obesity may be a stronger predictor for severe disease than BMI alone (BMI was ranked 13th by ShapVal). As before, TDI and age were ranked among the top. For age, slightly unexpectedly, a U-shaped curve was observed, which suggests lowest risk at the age group of 65-70. Note that model C may also capture RFs related to susceptibility to infection. It is possible, for instance, that younger individuals had higher risks of exposure due to work or social interactions. Among the top 10, two are related to general multiple comorbidities (cnt_tx and cnt_noncancer). Increased cystatin $\mathrm{C}$ and lower apolipoprotein $\mathrm{A}$ were also associated with higher susceptibility to severe infections, and HT and T2DM were also among the top 10. Considering PermImp as the ranking criteria, COPD, depression, and dementia were observed to have the lowest permutation $P$ values $(P=.002)$ though not top listed by ShapVal.

The interaction plot (Figure 4) shows WHR may interact with age, with elderly individuals showing more prominent effects from changes in WHR.

\section{Model D (Fatal Cases vs Population With No Known Infection)}

Based on ShapVal, age was the top feature, followed by TDI, WHR, number of drugs taken, and WC. Other top features included cystatin $\mathrm{C}$, testosterone, HT, RDW, and pulse rate. Higher levels of these features (or presence of comorbidity) generally lead to higher mortality risks. Based on PermImp, T2DM, dementia, and COPD were the most highly ranked (ignoring features that are already listed in the top 10 by ShapVal).

Shapley interaction analysis suggested that the top interacting pairs involved age and some of the top contributing features (Figure 4 and Figure S8 in Multimedia Appendix 3). The effects of testosterone (likely also reflects gender effects) and TDI were more prominent among the elderly, while the effect of BMI was larger in the younger age groups.

As for important variables from the sex-stratified analysis, the top variables were similar which included age, WC/WHR, cystatin $\mathrm{C}$, number of medications received, socioeconomic status (as reflected by TDI), among others (Table S3c in Multimedia Appendix 1).

\section{PermImp Compared With ShapVal}

Overall speaking, the PermImp measure tends to rank binary traits higher than ShapVal. Of note, several diseases were consistently top listed by PermImp across the 4 cohorts (though some were not highlighted by ShapVal), including CAD, atrial fibrillation (AF), T2DM, and dementia, which were among the top 10 in at least three cohorts in Table 4. Other diseases that were listed at least twice included depression, COPD, stroke, and heart failure.

\section{Results From the "Lite" Model}

Here we highlight top contributing features for the "lite" models consisting of 27 predictors (Table S3b in Multimedia Appendix 1). Remarkably, the top 3 features (ranked by ShapVal) were consistent across all 4 cohorts. These features included age, cnt_tx, and WC (WHR was not included in the lite model as $\mathrm{WC}$ is easier to measure). Of note, sex and T2DM were ranked among the top 6 across all cohorts.

If we consider PermImp as the ranking criteria (further ranked by ShapVal if PermImp is equal), age, cnt_tx, and WC were still highly ranked and listed among the top 5 in at least three cohorts (Table S3b in Multimedia Appendix 1). T2DM was ranked among the top 5 in all cohorts. Other potential risk factors included dementia (top 10 across 3 cohorts) as well as AF, COPD, and CAD (top 10 across 2 cohorts).

\section{Results From the Logistic Model}

As discussed above, we primarily focused on the XGboost ML model as it can capture nonlinear relationships and interactions between predictors. Here we also performed our analyses with logistic regression (LR) for comparison. For prediction performance (Table S7a in Multimedia Appendix 1), the AUC-ROC of the full LR model was 0.728 (95\% CI $0.715-0.741), 0.810$ (95\% CI $0.786-0.834), 0.712$ (95\% CI $0.701-0.724)$, and 0.833 (95\% CI 0.810-0.856), respectively, for cohorts A-D. For the "lite" model (using 27 predictors only), the AUC-ROC of the LR approach was 0.722 (95\% CI $0.709-0.735), 0.824$ (95\% CI 0.801-0.848), 0.697 (95\% CI $0.685-0.709$ ), and 0.834 (95\% CI 0.812-0.857), respectively, for cohorts A-D (Table S7a in Multimedia Appendix 1). These figures were very close to those obtained by XGboost, although AUC-ROC using LR was slightly higher in general (median difference $=0.005$ ). If we compute the $R R$ of individuals at the highest and lowest $\mathrm{k} \%$ of predicted risks, the results were 
generally similar (Table S7b in Multimedia Appendix 1). For cohort D and the full model of cohort B, XGboost performed better than LR at the extreme ends of predicted risks, with observed risk $=0$ (ie, no cases were observed) for those predicted at the lowest $5 \%$ of risk (Table 2).

While prediction is one of our goals, uncovering important contributing factors and their relationship to COVID-19 severity is a major objective of this study. In fact, the latter is considered our primary objective when considering the analyses within patients infected (cohorts A and B). As LR assumes linearity on a log-odds scale, it could not capture nonlinear relationships or "threshold effects" of variables on disease severity.

\section{Individual Shapley Decision Plots and Online Calculator}

We also showed individual Shapley decision plot for 3 individuals with the highest, median, and lowest predicted risks in each cohort (Figure S10 in Multimedia Appendix 3). The $\mathrm{y}$-axis is based on a log-odds scale.

To facilitate further research and studies on risk-prediction models, we also constructed an online risk calculation tool (for "lite" model) [53]. The online tool can also construct a Shapley decision plot based on individual risk factors.

\section{Discussion}

\section{Principal Findings}

In this study we have performed 4 sets of analysis, predicting severe or fatal COVID-19 infection among affected individuals or in the population. We observed good predictive power from the XGboost ML models, especially for the prediction of mortality. We also identified risk factors for increased severity or mortality, and uncovered possible nonlinear effects of some features, which may be clinically relevant and shed light on disease mechanisms.

\section{Prediction of Severity/Mortality}

In general, our prediction models achieved reasonably good predictive power. The models predicted mortality (AUC $81 \%-83 \%$ ) better than severity of disease. As discussed earlier, in the absence of better alternatives, hospitalization (test performed as inpatient) was used as a proxy for severity. However, reasons or criteria for hospitalization may vary across individuals or hospitals, and some tests may be performed in inpatients for surveillance or due to other confirmed/suspected cases in the ward. As a result, hospitalized patients could also include some with mild or moderate illnesses, which may also impair the prediction performance. By contrast, mortality from infection is a more objective outcome. Other studies (eg, [54-56]) have also defined "severe" or "critical" disease based on intensive care unit admission or need for ventilatory support. However, we could not find sufficiently detailed clinical data to support such a classification at the time of this analysis.

\section{Discriminatory Power of the Models and Clinical Implications}

By assessing the proportion of cases explained by those at the top $\mathrm{k} \%$ of predicted risks, we observed in general a strong enrichment of cases among those with high predicted risks, indicating good discriminative ability of the models and suggesting the possibility to focus on the highest-risk group for targeted preventions or treatment. A similar strong enrichment was also observed for the lite model with fewer predictors. We also observed large RRs of the actual outcomes when comparing individuals at high and low percentiles of predicted risks. For example, for the prediction of mortality among the infected, the RR was up to 158 times ( $20 \%$ vs $0.1 \%$ ) when comparing and top and bottom deciles using the full model, and 28.38 times when considering the simplified model ( $21 \%$ vs $0.8 \%)$. These results suggest that the prediction models may be used for risk stratification and prioritizing those at higher risks of deterioration, for early medical attention or admission. As the "lite" model only relies on demographic data and information on comorbidities, risk stratification may be conducted even at the start of the illness without other blood or imaging results.

\section{Previous Relevant Works}

A number of studies have focused on prediction of severity/mortality of COVID-19 (corresponding to our prediction in cohorts $\mathrm{A}$ and $\mathrm{B}$ ) and were reviewed in [8]. For cohort A (prediction of severity among infected), the AUC is $72.3 \%$, which is moderate but not as good as many previous ML models for severity prediction [8]. The AUC for prediction of mortality is much higher (AUC $=81.4 \%$ ), although we noted that some studies have reported higher predictive power from clinical symptoms, blood biochemistry on admission, and imaging features [8]. We understand that without access to the above features, predictive performance may be inferior. By contrast, due to heterogeneity of clinical samples, treatment approaches, model evaluation methods, and other features across studies, direct comparisons of predictive performance across studies may be difficult. Here we are not aiming at deriving a highly accurate prediction model; the main purpose is to identify general or "baseline" risk factors for severe disease, thereby gaining insight into disease pathophysiology. However, we also showed that such clinical features or blood measurements, even when collected much earlier in time, may still be highly predictive of outcomes and hence may be incorporated into existing prediction algorithms. The models here may also be useful when blood results or imaging are not available (eg, before admission) and the goal is to quickly classify a patient's risk.

For cohorts $\mathrm{C}$ and $\mathrm{D}$, the general population (with no known infection) was treated as "controls." Compared with cohorts A and $\mathrm{B}$, the identified risk factors may also increase the overall susceptibility to infection. The AUC for cohort C (severe/fatal disease) is about $70 \%$ but is much higher when mortality is considered as the outcome ( $\mathrm{AUC}=\sim 83 \%$ ). To our knowledge, there are still very few predictive models built at a generalpopulationlevel to identify susceptible individuals; this work is among the first to employ an ML approach to predict the risk of COVID-19/severe infection at a population level. DeCaprio et al [57] proposed an ML model to assess the vulnerability to COVID-19 in the population. However, due to limited data, no actual COVID-19 patients were included and "proxy" outcomes were used instead. Models were built from mainly demographic and comorbidity data to predict 
hospitalization due to acute respiratory distress syndrome, pneumonia, influenza, acute bronchitis, and other respiratory tract infections.

Another very recent study ("QCOVID" study) from the UK [58] utilized general practice records from 6.08 million adults (age 19 to 100) as the derivation cohort and 2.17 million adults as the validation set. Mortality from COVID-19 was the primary outcome and a survival model (subdistribution hazard model) [58] was used to predict mortalities. The predictors included demographic (eg, age, TDI, ethnicity), lifestyle (eg, BMI, smoking), and a large range of comorbid conditions. The resulting Harrell's C (comparable to AUC) was 0.928. However, we note that the QCOVID study included individuals of a much younger age range (19 or older), which will improve predictive performance, as age is by far the most important predictor of mortality, with markedly reduced risks in younger individuals. For example, if we refer to age-specific predictive performance (see Supplementary Table C in the study [58]), Harrell's C for mortality was $0.678,0.831,0.812$, and 0.814 in 50-59, 60-69, 70-79, and 80+ year olds, respectively, for males in the first follow-up period (January 24 to April 30, 2020). For females, the corresponding numbers were $0.618,0.77,0.866$, and 0.821 . These numbers reflect lower predictive power when restricted to a narrower age range. One main difference between this work and the above study is that we employed an XGboost $M L$ approach which is able to also capture nonlinear and more complex interaction effects. As shown in our Shapley dependence plots, the models were able to reveal nonlinear effects in a data-driven manner. We also included a number of blood measurements to shed light on potential new risk factors and mechanisms underlying the disease. The QCOVID study employed a survival model (subdistribution hazard) that accounts for time-to-event and competing risks; however, the proportional hazards assumption is required which may not hold due to restrictions/interventions introduced during the period (ie, time-dependent associations may be present).

A few other studies have investigated risk factors (especially comorbidities) for COVID-19 infection in the UKBB. For example, Atkins et al [12] studied elderly individuals (age >65) in UKBB, and found that HT, history of falls, CAD, T2DM, and asthma were the top comorbidities among hospitalized cases. The analysis was restricted to the elderly population, however. In a more recent work, McQueenie et al [13] studied the impact of multiple comorbidities and polypharmacy on infection risks. Having 2 or more long-term conditions, cardiometabolic disorders, and polypharmacy were associated with heightened risks of infection. Among individuals with multiple comorbidities, severe obesity and IRF may lead to increased risks. Another study of primary care patients in the UK revealed that deprivation, male sex, older age, ethnicity (being Black), and chronic renal disease were associated with higher risks of being tested positive [59]. Another large-scale British primary care study of more than 17 million individuals revealed similar risk factors as above [60]. There is also a relatively large literature on the study of risk factors associated with severe or fatal disease [15-18,61-64]. Some commonly reported risk factors included age, sex, obesity, T2DM, HT, renal, cardiometabolic, and respiratory disorders. As discussed above, an important difference between the above epidemiological studies and this work is that we employed XGboost, an ML approach that can uncover nonlinear and interaction effects, while other studies mostly employed regression models that assume linear and additive effects of covariates. We also performed a comprehensive analysis including 4 models covering different outcomes and both infected and population cohorts.

\section{Comparison With Logistic Model}

We have performed LR to compare with XGboost on cohorts $\mathrm{A}$ and $\mathrm{B}$. The differences in predictive performance appeared to be small. The number of cases (especially fatalities) is relatively small in this data set, and this may limit the predictive performance of more complex models such as XGboost, which may be expected to improve with larger case numbers. An important advantage of XGboost is that it can detect nonlinear relationships when compared with LR. In addition, XGboost may handle multiple collinearity better than LR. Assuming 2 highly correlated features $\mathrm{A}$ and $\mathrm{B}$, for each specific tree usually only 1 variable will be used and as the trees are sequential, the focus of the model will be usually on one but not on both features [65]. Hence, XGboost also handles multicollinearity well, which is important here as many clinical variables are correlated. XGboost also directly models interaction between variables. It is much more difficult for LR to model interactions due to the rapid increase in feature space when interaction terms are included.

\section{Highlights of Potential Risk Factors}

For the limit of space, we shall only highlight the top 5-10 risk factors ranked by ShapVal here. Across the 4 cohorts, age and cardiometabolic risk factors predominate the top risk factors. Age and WHR/WC were ranked among the top 5 across all 4 cohorts. The number of medications taken was among the top 5 across all cohorts, and cystatin $\mathrm{C}$ (reflecting renal function) was among the top 10 across all cohorts. HbA1c was a top 10 risk factor for cohort $\mathrm{A}$, and T2DM was also highly ranked across multiple cohorts especially when PermImp was considered. TDI (reflecting socioeconomic status) was among the top 10 in most cohorts. As described above, results from the "lite" models were generally in line with those from the full models, with age, WC, and cnt_tx consistently ranked as the top 3 .

Obesity has been observed to be a major risk factor for susceptibility or severity of infection in the UKBB $[14,66]$ and in many other studies $[67,68]$. The observation that WC/WHR were highly ranked suggests that central obesity is a major risk factor and may be a better predictor of severity than BMI alone.

Another major risk factor we identified is IRF, as reflected by elevated risks with raised urea and cystatin C. Several studies also suggested that IRF increases risk of mortality [64,69,70], although it is probably not as widely recognized as cardiometabolic disorders as a major risk factor. Because COVID-19 itself may lead to renal failure, our findings specifically suggest that underlying or baseline IRF is an important risk factor. The high ranking of cystatin $\mathrm{C}$ also indicates that this measure may better reflect renal function than 
urea or creatinine (which were also included in our analysis) $[71,72]$, and may serve as a superior predictor for COVID-19 severity.

Other potential risk factors briefly highlighted below were less reported. As some were listed only once or twice among the top 10, and for some their ShapVal was close to other risk factors, further replications are required. For example, testosterone was top ranked by XGboost (for mortality), with higher levels associated with increased risk. This may partially reflect that males are at a higher risk of fatal infections, but it remains to be studied whether testosterone itself is involved in the pathophysiology of severe COVID-19, as the ML model chose this variable instead of sex. Studies have suggested that elevated or reduced testosterone levels may be associated with a more severe clinical course [73]. Besides, interestingly, 5-alpha-reductase inhibitors or androgen-deprivation therapy has been shown to be associated with a lower risk or severity of disease [74,75]. We also found a few hematological indices that may be potential risk factors. High RDW was associated with mortality in our study and was also identified in a recent meta-analysis of 3 studies as a risk factor [76]. Low lymphocyte percentage was a top 10 risk factor in cohort $\mathrm{B}$, which may be related to immune functioning and response to infections. Lymphopenia was reported as a main hematological finding in those with severe illnesses [40,77]. Most previous studies considered hematological indices at admission or during hospitalization. Slightly surprisingly, this study suggested that high RDW or reduced lymphocyte percentage prior to the diagnosis may also be predictive of worse outcomes.

\section{Comorbid Diseases Associated With Severity as Highlighted by PermImp}

Among the diseases being included as covariates, T2DM is most consistently ranked among the top, no matter whether full or lite models are used, and regardless of ranking by ShapVal or PermImp ( $P$ value). T2DM has been shown in numerous studies to be associated with higher risk and severity of infection $[78,79]$. We noted some discrepancy between the ranked results based on ShapVal and those based on PermImp. In general, the latter measure favors binary variable, while ShapVal alone tends to rank continuous variables higher. We are unsure about the exact reason, but it may be an interesting topic for further methodology studies. If we employed a composite ranking criteria based on PermImp followed by ShapVal, then a few more diseases were ranked among the top 10, such as hypertension and COPD. For cohort D, T2DM, dementia, COPD, AF, heart failure, and CAD were also top ranked, suggesting that a range of chronic cardiovascular, respiratory, and neuropsychiatric conditions may be associated with increased mortality.

\section{Full and Lite Prediction Models}

We note that the simplified (lite) prediction model has very similar predictive performance (as assessed by AUC) to the "full" model with a larger panel of predictors. However, it is important to note that features associated with the outcome may not always improve predictive power. AUC is relatively insensitive to detecting changes in predictive performance when additional risk factors are added [80-82].
For example, Pencina et al [80] showed that in the prediction of cardiovascular disease risk in a study on women's health, adding extra established risk factors often result in minimal improvements in AUC. For instance, in a model with age, SBP, and smoking, adding any lipid measures result in only an increase of 0.01 in AUC from the baseline of 0.76. In the same vein, starting from a full prediction model [containing Ln(age), Ln(SBP), smoking, Ln(Total cholesterol), Ln(HDL)], deleting any one of these established risk factors (except age) resulted in a very small reduction in AUC of $<0.02$. In general, for a model with high baseline AUC from existing predictors (eg, age, sex, and obesity in the case of COVID-19), including additional predictors may not result in much improvement in discriminative power or AUC [83].

Nevertheless, it is still valuable to study variable importance (eg, ShapVal) from the ML model as it may shed light on the pathophysiology of the disease. For example, many factors such as age and T2DM may lead to poorer renal function (and higher cystatin C), which in turn may increase the severity of infection. Given that age, T2DM, and other main comorbidities are already modeled, adding cystatin $\mathrm{C}$ may not improve discriminative power of the model. However, its inclusion may still change the predicted probability of outcome, which will be reflected in ShapVal. The high ranking of cystatin C (based on ShapVal) may shed light on renal impairment as a potential mechanism associated with clinical deterioration.

Some limitations have been discussed above; for example, the use of hospitalization as a proxy for severity, and that the predictors were recorded prior to the pandemic. We briefly discuss other limitations here. The UKBB is a very large-scale study with detailed phenotypic data, but still the number of fatal cases is relatively small. In addition, the UKBB is not entirely representative of the UK population, as participants tend to be healthier and wealthier overall [84]. Further, it remains to be studied whether the findings are generalizable to other populations. Symptom measures and lung imaging features were not available at the time of analysis. Despite adjusting for a rich set of predictors and that all predictors were recorded prior to the outbreak, causality cannot be confirmed from this study, due to risk of residual confounding by unknown factors. This study was performed on a cohort with age over 50, and generalizability to younger individuals remains to be studied. In cohorts $\mathrm{C}$ and $\mathrm{D}$, the population with no known infection was regarded as controls. It is expected that some may become infected in the future, and some may have been infected but not tested; however, the chance of missing cases of severe infection is probably not high. Since the UKBB represents a relatively healthy population with a low rate of severe COVID-19 cases so far $(236 / 468,114,0.50 \%)$, we expect the use of "unscreened" controls is unlikely to result in substantial bias.

Regarding the ML model, XGboost is a state-of-the-art method that has been consistently shown to be the best or one of the best ML methods in supervised learning tasks/competitions [85] (especially for tasks not involving computer vision or natural language processing). Nevertheless, other ML methods may still be useful or may uncover novel risk factors. Assessing variable importance is a long-standing problem in ML; here we 
mainly employed ShapVal, which is both computationally fast and was shown to have good theoretical properties [46,47].

\section{Conclusions}

In conclusion, we identified a number of baseline risk factors for severe/fatal infection by an ML approach. Shapley dependence plots revealed possible nonlinear and "threshold" effects of risk factors on the risks of infection or severity. To summarize, age, central obesity, IRF, multiple comorbidities, cardiometabolic abnormalities or disorders (especially T2DM), and low socioeconomic status may predispose to poorer outcomes, among other risk factors. The prediction models (of cohorts C/D) may be useful at a population level to identify those susceptible to developing severe/fatal infections, thereby facilitating targeted prevention strategies. Further replication and validation in independent cohorts are required to confirm our findings.

\section{Acknowledgments}

This work was supported partially by the Lo Kwee Seong Biomedical Research Fund from The Chinese University of Hong Kong, the KIZ-CUHK Joint Laboratory of Bioresources and Molecular Research of Common Diseases, the Kunming Institute of Zoology, and The Chinese University of Hong Kong. We thank Professor Pak Sham for support on data access and analyses, and Ms Qiu Jinghong for formatting and editing of the manuscript.

\section{Authors' Contributions}

$\mathrm{H}-\mathrm{CS}$ contributed to the conception and design, as well as supervised the study; H-CS and KC-YW were responsible for the analytic methodology. KC-YW (main), YX, LY, and H-CS performed data analysis. H-CS and KC-YW performed data interpretation. H-CS, with input from $\mathrm{KC}-\mathrm{YW}$, drafted the manuscript.

\section{Conflicts of Interest}

None declared.

\section{Multimedia Appendix 1}

Supplementary tables S1-S8.

[XLSX File (Microsoft Excel File), 2018 KB-Multimedia Appendix 1]

\section{Multimedia Appendix 2}

Simulation experiment to verify the validity of permutation testing for rare outcomes.

[DOCX File, 16 KB-Multimedia Appendix 2]

\section{Multimedia Appendix 3}

Supplementary figures.

[XLSX File (Microsoft Excel File), 14559 KB-Multimedia Appendix 3]

\section{References}

1. Li Q, Guan X, Wu P, Wang X, Zhou L, Tong Y, et al. Early Transmission Dynamics in Wuhan, China, of Novel Coronavirus-Infected Pneumonia. N Engl J Med 2020 Mar 26;382(13):1199-1207 [FREE Full text] [doi:

10.1056/NEJMoa2001316] [Medline: $\underline{\text { 31995857] }}$

2. Novel Coronavirus Pneumonia Emergency Response Epidemiology Team. [The epidemiological characteristics of an outbreak of 2019 novel coronavirus diseases (COVID-19) in China]. Zhonghua Liu Xing Bing Xue Za Zhi 2020 Feb 17;41(2):145-151. [doi: 10.3760/cma.j.issn.0254-6450.2020.02.003] [Medline: $\underline{\text { 32064853] }}$

3. Guan W, Ni Z, Hu Y, Liang W, Ou C, He J, China Medical Treatment Expert Group for Covid-19. Clinical Characteristics of Coronavirus Disease 2019 in China. N Engl J Med 2020 Apr 30;382(18):1708-1720 [FREE Full text] [doi: 10.1056/NEJMoa2002032] [Medline: 32109013]

4. Johns Hopkins Coronavirus Resource Center. URL: https://coronavirus.jhu.edu/map.html [accessed 2021-06-19]

5. Bayat V, Phelps S, Ryono R, Lee C, Parekh H, Mewton J, et al. A SARS-CoV-2 Prediction Model from Standard Laboratory Tests. Clin Infect Dis 2020 Aug 12:ciaa1175 [FREE Full text] [doi: 10.1093/cid/ciaa1175] [Medline: $\underline{32785701]}$

6. Knight SR, Ho A, Pius R, Buchan I, Carson G, Drake TM, ISARIC4C investigators. Risk stratification of patients admitted to hospital with covid-19 using the ISARIC WHO Clinical Characterisation Protocol: development and validation of the 4C Mortality Score. BMJ 2020 Sep 09;370:m3339 [FREE Full text] [doi: 10.1136/bmj.m3339] [Medline: $\underline{32907855]}$

7. Chung H, Ko H, Kang WS, Kim KW, Lee H, Park C, et al. Prediction and Feature Importance Analysis for Severity of COVID-19 in South Korea Using Artificial Intelligence: Model Development and Validation. J Med Internet Res 2021 Apr 19;23(4):e27060 [FREE Full text] [doi: 10.2196/27060] [Medline: 33764883] 
8. Wynants L, Van Calster B, Collins GS, Riley RD, Heinze G, Schuit E, et al. Prediction models for diagnosis and prognosis of covid-19: systematic review and critical appraisal. BMJ 2020 Apr 07;369:m1328 [FREE Full text] [doi: 10.1136/bmj.m1328] [Medline: $\underline{\text { 32265220] }}$

9. Alballa N, Al-Turaiki I. Machine learning approaches in COVID-19 diagnosis, mortality, and severity risk prediction: A review. Inform Med Unlocked 2021;24:100564 [FREE Full text] [doi: 10.1016/j.imu.2021.100564] [Medline: 33842685]

10. Adamidi ES, Mitsis K, Nikita KS. Artificial intelligence in clinical care amidst COVID-19 pandemic: A systematic review. Comput Struct Biotechnol J 2021;19:2833-2850 [FREE Full text] [doi: 10.1016/j.csbj.2021.05.010] [Medline: 34025952]

11. Vaid A, Somani S, Russak AJ, De Freitas JK, Chaudhry FF, Paranjpe I, et al. Machine Learning to Predict Mortality and Critical Events in a Cohort of Patients With COVID-19 in New York City: Model Development and Validation. J Med Internet Res 2020 Nov 06;22(11):e24018 [FREE Full text] [doi: 10.2196/24018] [Medline: 33027032]

12. Atkins J, Masoli J, Delgado J, Pilling L, Kuo C, Kuchel G, et al. Preexisting Comorbidities Predicting COVID-19 and Mortality in the UK Biobank Community Cohort. J Gerontol A Biol Sci Med Sci 2020 Oct 15;75(11):2224-2230 [REE Full text] [doi: 10.1093/gerona/glaa183] [Medline: 32687551]

13. McQueenie R, Foster HME, Jani BD, Katikireddi SV, Sattar N, Pell JP, et al. Correction: Multimorbidity, polypharmacy, and COVID-19 infection within the UK Biobank cohort. PLoS One 2021 May 6;16(5):e0251613 [REEE Full text] [doi: 10.1371/journal.pone.0251613] [Medline: 33956868]

14. Yates T, Razieh C, Zaccardi F, Davies MJ, Khunti K. Obesity and risk of COVID-19: analysis of UK biobank. Prim Care Diabetes 2020 Oct;14(5):566-567 [FREE Full text] [doi: 10.1016/j.pcd.2020.05.011] [Medline: $\underline{32493608]}$

15. Rod JE, Oviedo-Trespalacios O, Cortes-Ramirez J. A brief-review of the risk factors for covid-19 severity. Rev Saude Publica 2020;54:60 [FREE Full text] [doi: 10.11606/s1518-8787.2020054002481] [Medline: $\underline{32491116]}$

16. Romero Starke K, Petereit-Haack G, Schubert M, Kämpf D, Schliebner A, Hegewald J, et al. The Age-Related Risk of Severe Outcomes Due to COVID-19 Infection: A Rapid Review, Meta-Analysis, and Meta-Regression. Int J Environ Res Public Health 2020 Aug 17;17(16):5974 [FREE Full text] [doi: 10.3390/ijerph17165974] [Medline: $\underline{\text { 32824596] }}$

17. Wingert A, Pillay J, Gates M, Guitard S, Rahman S, Beck A, et al. Risk factors for severity of COVID-19: a rapid review to inform vaccine prioritisation in Canada. BMJ Open 2021 May 13;11(5):e044684 [FREE Full text] [doi: 10.1136/bmjopen-2020-044684] [Medline: 33986052]

18. Wolff D, Nee S, Hickey NS, Marschollek M. Risk factors for Covid-19 severity and fatality: a structured literature review. Infection 2021 Feb 28;49(1):15-28 [FREE Full text] [doi: 10.1007/s15010-020-01509-1] [Medline: 32860214]

19. Sudlow C, Gallacher J, Allen N, Beral V, Burton P, Danesh J, et al. UK biobank: an open access resource for identifying the causes of a wide range of complex diseases of middle and old age. PLoS Med 2015 Mar;12(3):e1001779 [FREE Full text] [doi: 10.1371/journal.pmed.1001779] [Medline: 25826379]

20. Data for COVID-19 research. URL: http://biobank.ndph.ox.ac.uk/showcase/exinfo.cgi?src=COVID19 [accessed 2021-09-08]

21. The official UK government website for data and insights on coronavirus (COVID-19). URL: https://coronavirus.data.gov.uk/ [accessed 2021-09-08]

22. Lopez Bernal J, Andrews N, Gower C, Robertson C, Stowe J, Tessier E, et al. Effectiveness of the Pfizer-BioNTech and Oxford-AstraZeneca vaccines on covid-19 related symptoms, hospital admissions, and mortality in older adults in England: test negative case-control study. BMJ 2021 May 13;373:n1088 [FREE Full text] [doi: 10.1136/bmj.n1088] [Medline: $\underline{33985964]}$

23. Tenforde M, Olson SM, Self WH, Talbot HK, Lindsell CJ, Steingrub JS, HAIVEN Investigators. Effectiveness of Pfizer-BioNTech and Moderna Vaccines Against COVID-19 Among Hospitalized Adults Aged $\geq 65$ Years - United States, January-March 2021. MMWR Morb Mortal Wkly Rep 2021 May 07;70(18):674-679 [FREE Full text] [doi: 10.15585/mmwr.mm7018e1] [Medline: $\underline{\text { 33956782] }}$

24. Dagan N, Barda N, Kepten E, Miron O, Perchik S, Katz MA, et al. BNT162b2 mRNA Covid-19 Vaccine in a Nationwide Mass Vaccination Setting. N Engl J Med 2021 Apr 15;384(15):1412-1423 [FREE Full text] [doi: 10.1056/NEJMoa2101765] [Medline: $\underline{33626250]}$

25. Haas EJ, Angulo FJ, McLaughlin JM, Anis E, Singer SR, Khan F, et al. Impact and effectiveness of mRNA BNT162b2 vaccine against SARS-CoV-2 infections and COVID-19 cases, hospitalisations, and deaths following a nationwide vaccination campaign in Israel: an observational study using national surveillance data. Lancet 2021 May 15;397(10287):1819-1829 [FREE Full text] [doi: 10.1016/S0140-6736(21)00947-8] [Medline: 33964222]

26. Dashti H, Roche EC, Bates DW, Mora S, Demler O. SARS2 simplified scores to estimate risk of hospitalization and death among patients with COVID-19. Sci Rep 2021 Mar 02;11(1):4945 [FREE Full text] [doi: 10.1038/s41598-021-84603-0] [Medline: 33654180]

27. Karaismailoglu E, Karaismailoglu S. Two novel nomograms for predicting the risk of hospitalization or mortality due to COVID-19 by the naïve Bayesian classifier method. J Med Virol 2021 May;93(5):3194-3201 [FREE Full text] [doi: 10.1002/jmv.26890] [Medline: 33599308]

28. Hamer M, Kivimäki M, Gale C, Batty G. Lifestyle risk factors, inflammatory mechanisms, and COVID-19 hospitalization: A community-based cohort study of 387,109 adults in UK. Brain Behav Immun 2020 Jul;87:184-187 [FREE Full text] [doi: 10.1016/j.bbi.2020.05.059] [Medline: 32454138] 
29. Pairo-Castineira E, Clohisey S, Klaric L, Bretherick AD, Rawlik K, Pasko D, GenOMICC Investigators, ISARIC4C Investigators, COVID-19 Human Genetics Initiative, 23andMe Investigators, BRACOVID Investigators, Gen-COVID Investigators, et al. Genetic mechanisms of critical illness in COVID-19. Nature 2021 Mar 11;591(7848):92-98. [doi: 10.1038/s41586-020-03065-y] [Medline: 33307546]

30. Walsh K, Jordan K, Clyne B, Rohde D, Drummond L, Byrne P, et al. SARS-CoV-2 detection, viral load and infectivity over the course of an infection. J Infect 2020 Sep;81(3):357-371 [FREE Full text] [doi: 10.1016/j.jinf.2020.06.067] [Medline: 32615199]

31. COVID-19 test results data. URL: http://biobank.ndph.ox.ac.uk/showcase/exinfo.cgi?src=COVID19 tests [accessed 2021-09-08]

32. Lever J, Krzywinski M, Altman N. Model selection and overfitting. Nat Methods 2016 Aug 30;13(9):703-704. [doi: 10.1038/nmeth.3968]

33. Stekhoven DJ, Bühlmann P. MissForest--non-parametric missing value imputation for mixed-type data. Bioinformatics 2012 Jan 01;28(1):112-118. [doi: 10.1093/bioinformatics/btr597] [Medline: 22039212]

34. Waljee AK, Mukherjee A, Singal AG, Zhang Y, Warren J, Balis U, et al. Comparison of imputation methods for missing laboratory data in medicine. BMJ Open 2013 Aug 01;3(8):e002847 [FREE Full text] [doi: 10.1136/bmjopen-2013-002847] [Medline: 23906948]

35. Shah A, Bartlett J, Carpenter J, Nicholas O, Hemingway H. Comparison of random forest and parametric imputation models for imputing missing data using MICE: a CALIBER study. Am J Epidemiol 2014 Mar 15;179(6):764-774 [FREE Full text] [doi: 10.1093/aje/kwt312] [Medline: 24589914]

36. Penone C, Davidson A, Shoemaker K, Di Marco M, Rondinini C, Brooks T, et al. Imputation of missing data in life-history trait datasets: which approach performs the best? Methods Ecol Evol 2014 Aug 08;5(9):961-970 [FREE Full text] [doi: 10.1111/2041-210x.12232]

37. Crowson CS, Atkinson EJ, Therneau TM. Assessing calibration of prognostic risk scores. Stat Methods Med Res 2016 Aug;25(4):1692-1706 [FREE Full text] [doi: 10.1177/0962280213497434] [Medline: 23907781]

38. Nixon J, Dusenberry M, Zhang L, Jerfel G, Tran D. Measuring Calibration in Deep Learning. CVPR Workshops. 2019. URL: https://openaccess.thecvf.com/content CVPRW 2019/html/Uncertainty and Robustness in Deep Visual Learning/ Nixon Measuring Calibration in Deep Learning CVPRW 2019 paper.html [accessed 2021-09-14]

39. Demler OV, Paynter NP, Cook NR. Tests of calibration and goodness-of-fit in the survival setting. Stat Med 2015 May 10;34(10):1659-1680 [FREE Full text] [doi: 10.1002/sim.6428] [Medline: 25684707]

40. Tan L, Wang Q, Zhang D, Ding J, Huang Q, Tang Y, et al. Correction: Lymphopenia predicts disease severity of COVID-19: a descriptive and predictive study. Signal Transduct Target Ther 2020 Apr 29;5(1):61 [FREE Full text] [doi: 10.1038/s41392-020-0159-1] [Medline: $\underline{33911071]}$

41. Song H, Diethe T, Kull M, Flach P. Distribution calibration for regression. 2019 Presented at: Proceedings of the 36th International Conference on Machine Learning; June 10-15, 2019; Long Beach, CA p. 5897-5906.

42. Niculescu-Mizil A, Caruana R. Predicting good probabilities with supervised learning. New York, NY: Association for Computing Machinery; 2005 Presented at: Proceedings of the 22nd international conference on Machine learning; August 7-11, 2005; Bonn, Germany p. 625-632. [doi: 10.1145/1102351.1102430]

43. Jiang X, Osl M, Kim J, Ohno-Machado L. Smooth isotonic regression: a new method to calibrate predictive models. AMIA Jt Summits Transl Sci Proc 2011;2011:16-20 [FREE Full text] [Medline: 22211175]

44. Steyerberg EW, Vergouwe Y. Towards better clinical prediction models: seven steps for development and an ABCD for validation. Eur Heart J 2014 Aug 01;35(29):1925-1931 [FREE Full text] [doi: 10.1093/eurheartj/ehu207] [Medline: 24898551]

45. Niculescu-Mizil A, Caruana R. Obtaining Calibrated Probabilities from Boosting. 2005 Presented at: 21st Conference on Uncertainty in Artificial Intelligence (UAI); July 26-29, 2005; Edinburgh, Scotland p. 413-420.

46. Lundberg S, Lee S. A unified approach to interpreting model predictions. Advances in neural information processing systems. In: Proceedings of the 31st International Conference on Neural Information Processing Systems. Red Hook, NY: Curran Associates; 2017 Presented at: 31st International Conference on Neural Information Processing Systems; December 4-9, 2017; Long Beach, CA p. 4768-4777.

47. Lundberg SM, Erion G, Chen H, DeGrave A, Prutkin JM, Nair B, et al. From Local Explanations to Global Understanding with Explainable AI for Trees. Nat Mach Intell 2020 Jan;2(1):56-67 [FREE Full text] [doi: 10.1038/s42256-019-0138-9] [Medline: 32607472]

48. Lundberg S, Erion G, Lee SI. Consistent individualized feature attribution for tree ensembles. arXiv 2018 [FREE Full text]

49. Altmann A, Toloşi L, Sander O, Lengauer T. Permutation importance: a corrected feature importance measure. Bioinformatics 2010 May 15;26(10):1340-1347. [doi: 10.1093/bioinformatics/btq134] [Medline: 20385727]

50. Witten DM, Tibshirani R. A framework for feature selection in clustering. J Am Stat Assoc 2010 Jun 01;105(490):713-726 [FREE Full text] [doi: 10.1198/jasa.2010.tm09415] [Medline: 20811510]

51. Tibshirani R, Walther G, Hastie T. Estimating the number of clusters in a data set via the gap statistic. Journal of the Royal Statistical Societyries B (Statistical Methodology) 2001;63(2):411-423. [doi: 10.1111/1467-9868.00293] 
52. Boyd K, Eng K, Page C. Area under the Precision-Recall Curve: Point Estimates and Confidence Intervals. In: Machine Learning and Knowledge Discovery in Databases. Berlin, Germany: Springer; 2013:451-466.

53. Risk Estimation for Covid-19 - Online Prediction Tool. URL: https://labsocuhk.ddns.net:8890/covid19/ [accessed 2021-09-08]

54. Subudhi S, Verma A, Patel AB, Hardin CC, Khandekar MJ, Lee H, et al. Comparing machine learning algorithms for predicting ICU admission and mortality in COVID-19. NPJ Digit Med 2021 May 21;4(1):87 [FREE Full text] [doi: 10.1038/s41746-021-00456-x] [Medline: 34021235]

55. Patrício A, Costa RS, Henriques R. Predictability of COVID-19 Hospitalizations, Intensive Care Unit Admissions, and Respiratory Assistance in Portugal: Longitudinal Cohort Study. J Med Internet Res 2021 Apr 28;23(4):e26075 [FREE Full text] [doi: 10.2196/26075] [Medline: $\underline{33835931]}$

56. Yun K, Lee JS, Kim EY, Chandra H, Oh B, Oh J. Severe COVID-19 Illness: Risk Factors and Its Burden on Critical Care Resources. Front Med (Lausanne) 2020 Nov 19;7:583060 [FREE Full text] [doi: 10.3389/fmed.2020.583060] [Medline: $\underline{33330540]}$

57. DeCaprio D, Gartner J, McCall CJ, Burgess T, Garcia K, Kothari S, et al. Building a COVID-19 vulnerability index. J Med Artif Intell 2020 Dec;3:15-15. [doi: 10.21037/jmai-20-47]

58. Clift AK, Coupland CAC, Keogh RH, Diaz-Ordaz K, Williamson E, Harrison EM, et al. Living risk prediction algorithm (QCOVID) for risk of hospital admission and mortality from coronavirus 19 in adults: national derivation and validation cohort study. BMJ 2020 Oct 20;371:m3731 [FREE Full text] [doi: 10.1136/bmj.m3731] [Medline: 33082154]

59. de Lusignan S, Dorward J, Correa A, Jones N, Akinyemi O, Amirthalingam G, et al. Risk factors for SARS-CoV-2 among patients in the Oxford Royal College of General Practitioners Research and Surveillance Centre primary care network: a cross-sectional study. The Lancet Infectious Diseases 2020 Sep 07;20(9):1034-1042 [FREE Full text] [doi: 10.1016/S1473-3099(20)30371-6]

60. Williamson EJ, Walker AJ, Bhaskaran K, Bacon S, Bates C, Morton CE, et al. Factors associated with COVID-19-related death using OpenSAFELY. Nature 2020 Aug;584(7821):430-436. [doi: 10.1038/s41586-020-2521-4] [Medline: 32640463$]$

61. Noor FM, Islam MM. Prevalence and Associated Risk Factors of Mortality Among COVID-19 Patients: A Meta-Analysis. J Community Health 2020 Dec;45(6):1270-1282 [FREE Full text] [doi: 10.1007/s10900-020-00920-x] [Medline: 32918645]

62. Rahman A, Sathi N. Risk factors of the severity of COVID-19: A meta-analysis. Int J Clin Pract 2021 Jul;75(7):e13916 [FREE Full text] [doi: 10.1111/ijcp.13916] [Medline: 33372407]

63. Zhou Y, Chi J, Lv W, Wang Y. Obesity and diabetes as high-risk factors for severe coronavirus disease 2019 (Covid-19). Diabetes Metab Res Rev 2021 Feb 20;37(2):e3377 [FREE Full text] [doi: 10.1002/dmrr.3377] [Medline: 32588943]

64. Harrison SL, Fazio-Eynullayeva E, Lane DA, Underhill P, Lip GYH. Comorbidities associated with mortality in 31,461 adults with COVID-19 in the United States: A federated electronic medical record analysis. PLoS Med 2020 Sep 10;17(9):e1003321 [FREE Full text] [doi: 10.1371/journal.pmed.1003321] [Medline: 32911500]

65. Understand Your Dataset with Xgboost. XGBoost R Package. 2020. URL: https://xgboost.readthedocs.io/en/latest/R-package/ discoverYourData.html [accessed 2021-09-08]

66. Hamer M, Gale CR, Kivimäki M, Batty GD. Overweight, obesity, and risk of hospitalization for COVID-19: A community-based cohort study of adults in the United Kingdom. Proc Natl Acad Sci U S A 2020 Sep 01;117(35):21011-21013 [FREE Full text] [doi: 10.1073/pnas.2011086117] [Medline: 32788355]

67. Popkin BM, Du S, Green WD, Beck MA, Algaith T, Herbst CH, et al. Individuals with obesity and COVID-19: A global perspective on the epidemiology and biological relationships. Obes Rev 2020 Nov;21(11):e13128 [FREE Full text] [doi: 10.1111/obr.13128] [Medline: 32845580]

68. Tamara A, Tahapary DL. Obesity as a predictor for a poor prognosis of COVID-19: A systematic review. Diabetes Metab Syndr 2020;14(4):655-659 [FREE Full text] [doi: 10.1016/j.dsx.2020.05.020] [Medline: $\underline{32438328]}$

69. Di Castelnuovo A, Bonaccio M, Costanzo S, Gialluisi A, Antinori A, Berselli N, COvid-19 RISkTreatments (CORIST) collaboration. Common cardiovascular risk factors and in-hospital mortality in 3,894 patients with COVID-19: survival analysis and machine learning-based findings from the multicentre Italian CORIST Study. Nutr Metab Cardiovasc Dis 2020 Oct 30;30(11):1899-1913 [FREE Full text] [doi: 10.1016/j.numecd.2020.07.031] [Medline: 32912793]

70. Uribarri A, Núñez-Gil IJ, Aparisi A, Becerra-Muñoz VM, Feltes G, Trabattoni D, HOPE COVID-19 Investigators. Impact of renal function on admission in COVID-19 patients: an analysis of the international HOPE COVID-19 (Health Outcome Predictive Evaluation for COVID 19) Registry. J Nephrol 2020 Aug;33(4):737-745 [FREE Full text] [doi: 10.1007/s40620-020-00790-5] [Medline: $\underline{\text { 32602006] }}$

71. Hojs R, Bevc S, Ekart R, Gorenjak M, Puklavec L. Serum cystatin C as an endogenous marker of renal function in patients with mild to moderate impairment of kidney function. Nephrol Dial Transplant 2006 Jul;21(7):1855-1862. [doi: 10.1093/ndt/gfl073] [Medline: 16524933]

72. Shlipak MG, Mattes MD, Peralta CA. Update on cystatin C: incorporation into clinical practice. Am J Kidney Dis 2013 Sep;62(3):595-603 [FREE Full text] [doi: 10.1053/j.ajkd.2013.03.027] [Medline: 23701892]

73. Giagulli VA, Guastamacchia E, Magrone T, Jirillo E, Lisco G, De Pergola G, et al. Worse progression of COVID-19 in men: Is testosterone a key factor? Andrology 2021 Jan;9(1):53-64 [FREE Full text] [doi: 10.1111/andr.12836] [Medline: 32524732] 
74. Cadegiani F, McCoy J, Wambier C, Goren A. 5-Alpha-Reductase Inhibitors Reduce Remission Time of COVID-19: Results From a Randomized Double Blind Placebo Controlled Interventional Trial in 130 SARS-CoV-2 Positive Men. medRxiv 2020. [doi: 10.1101/2020.11.16.20232512]

75. Montopoli M, Zumerle S, Vettor R, Rugge M, Zorzi M, Catapano C, et al. Androgen-deprivation therapies for prostate cancer and risk of infection by SARS-CoV-2: a population-based study (N = 4532). Ann Oncol 2020 Aug;31(8):1040-1045 [FREE Full text] [doi: 10.1016/j.annonc.2020.04.479] [Medline: 32387456]

76. Lippi G, Henry B, Sanchis-Gomar F. Red Blood Cell Distribution Is a Significant Predictor of Severe Illness in Coronavirus Disease 2019. Acta Haematol 2021 Aug 25;144(4):360-364 [FREE Full text] [doi: 10.1159/000510914] [Medline: 32841949]

77. Terpos E, Ntanasis-Stathopoulos I, Elalamy I, Kastritis E, Sergentanis TN, Politou M, et al. Hematological findings and complications of COVID-19. Am J Hematol 2020 Jul;95(7):834-847 [FREE Full text] [doi: 10.1002/ajh.25829] [Medline: $\underline{32282949]}$

78. Gupta R, Hussain A, Misra A. Diabetes and COVID-19: evidence, current status and unanswered research questions. Eur J Clin Nutr 2020 Jun 13;74(6):864-870 [FREE Full text] [doi: 10.1038/s41430-020-0652-1] [Medline: 32404898]

79. Apicella M, Campopiano MC, Mantuano M, Mazoni L, Coppelli A, Del Prato S. COVID-19 in people with diabetes: understanding the reasons for worse outcomes. The Lancet Diabetes \& Endocrinology 2020 Sep;8(9):782-792. [doi: 10.1016/s2213-8587(20)30238-2]

80. Pencina M, D' Agostino R, D' Agostino R, Vasan R. Evaluating the added predictive ability of a new marker: From area under the ROC curve to reclassification and beyond. Statist. Med 2007 Jan 30;27(2):157-172 [FREE Full text] [doi: 10.1002/sim.2929]

81. Cook NR. Use and Misuse of the Receiver Operating Characteristic Curve in Risk Prediction. Circulation 2007 Feb 20;115(7):928-935. [doi: 10.1161/circulationaha.106.672402]

82. Ware JH. The limitations of risk factors as prognostic tools. N Engl J Med 2006 Dec 21;355(25):2615-2617. [doi: 10.1056/NEJMp068249] [Medline: 17182986]

83. Janssens A, Martens F. Reflection on modern methods: Revisiting the area under the ROC Curve. Int J Epidemiol 2020 Aug 01;49(4):1397-1403. [doi: 10.1093/ije/dyz274] [Medline: 31967640]

84. Fry A, Littlejohns T, Sudlow C, Doherty N, Adamska L, Sprosen T, et al. Comparison of Sociodemographic and Health-Related Characteristics of UK Biobank Participants With Those of the General Population. Am J Epidemiol 2017 Nov 01;186(9):1026-1034 [FREE Full text] [doi: 10.1093/aje/kwx246] [Medline: 28641372]

85. Nielsen D. Tree Boosting With XGBoost. Master thesis, Norwegian University of Science and Technology. 2016. URL: http://pzs.dstu.dp.ua/DataMining/boosting/bibl/Didrik.pdf [accessed 2021-09-08]

\author{
Abbreviations \\ AF: atrial fibrillation \\ AUC-PRC: area under the precision-recall curve \\ AUC-ROC: area under the receiving-operating characteristic curve \\ CAD: coronary artery disease \\ COPD: chronic obstructive pulmonary disease \\ ECE: expected calibration error \\ HDL: high-density lipoprotein \\ HT: hypertension \\ IRF: impaired renal function \\ LR: logistic regression \\ MCC: Matthews correlation coefficient \\ MCE: maximum calibration error \\ MICE: multiple imputation by chained equation \\ ML: machine learning \\ RDW: red blood cell distribution width \\ RF: risk factor \\ RR: relative risk \\ SBP: systolic blood pressure \\ T1DM: type 1 diabetes mellitus \\ T2DM: type 2 diabetes mellitus \\ TDI: Townsend deprivation index \\ UKBB: UK Biobank \\ WC: waist circumference \\ WHR: waist-to-hip ratio
}


Edited by Y Khader; submitted 12.04.21; peer-reviewed by D DeCapprio, T Lee, H Oyama; comments to author 12.05.21; revised version received 24.06.21; accepted 31.07.21; published 30.09.21

Please cite as:

Wong $\mathrm{KCY}$, Xiang $Y$, Yin L, So HC

Uncovering Clinical Risk Factors and Predicting Severe COVID-19 Cases Using UK Biobank Data: Machine Learning Approach JMIR Public Health Surveill 2021;7(9):e29544

URL: https://publichealth.jmir.org/2021/9/e29544 doi: $10.2196 / 29544$

PMID:

CKenneth Chi-Yin Wong, Yong Xiang, Liangying Yin, Hon-Cheong So. Originally published in JMIR Public Health and Surveillance (https://publichealth.jmir.org), 30.09.2021. This is an open-access article distributed under the terms of the Creative Commons Attribution License (https://creativecommons.org/licenses/by/4.0/), which permits unrestricted use, distribution, and reproduction in any medium, provided the original work, first published in JMIR Public Health and Surveillance, is properly cited. The complete bibliographic information, a link to the original publication on https://publichealth.jmir.org, as well as this copyright and license information must be included. 